\title{
Non-adiabatic effects in thermochemistry, spectroscopy and kinetics: the general importance of all three Born-Oppenheimer breakdown corrections
}

\author{
Jeffrey R. Reimers ${ }^{\text {ab*, Laura K. McKemmish, }}{ }^{\text {cd }}$ Ross H. McKenzie, ${ }^{e}$ and Noel S. Hush ${ }^{\text {df }}$ \\ 5 Received (in $X X X, X X X) X$ th $X X X X X X X X X 20 X X$, Accepted $X$ th $X X X X X X X X X 20 X X$ \\ DOI: $10.1039 / b 000000 x$
}

The three correction terms for Born-Oppenheimer (BO) breakdown, the adiabatic diagonal correction (DC), the first-derivative momentum non-adiabatic correction (FD), and the second-derivative kineticenergy non-adiabatic correction (SD), are shown to all contribute to thermodynamic and spectroscopic 10 properties as well as to thermal non-diabatic chemical reaction rates. While DC often accounts for $>80 \%$ of thermodynamic and spectroscopic property changes, the commonly used practice of including only the FD correction in kinetics calculations is rarely found to be adequate. For electron-transfer reactions not in the inverted region, the common physical picture that diabatic processes occur because of surface hopping from the ground-state $\mathrm{BO}$ surface to an excited state surface near the transition state and back again is 15 shown to be inadequate as the DC acts first to block access to the transition state by dramatically increasing the transition state energy from $\Delta E^{\dagger}=\left(E_{0}+\lambda\right)^{2} / 4 \lambda-|J|$ by $\Delta \Delta E^{\dagger}=(\hbar \omega)^{2} \lambda / 16 J^{2}$ (where $E_{0}$ is the free-energy change, $\lambda$ the reorganization energy, $J$ the electronic coupling and $\omega$ the vibration frequency). However, the rate constant in the weakly-coupled Golden-Rule limit is identified as being only inversely proportional to $\Delta \Delta E^{\dagger}$ rather than exponentially damped, owing to the effects of tunneling 20 and surface hoping. Such weakly-coupled long-range electron-transfer processes should therefore not be described as "non-adiabatic" processes as they may well be described simply and easily based on the Born-Huang ground-state adiabatic surfaces made by adding the DC to the BO surfaces; instead, they should be called just "non-Born-Oppenheimer" processes. These conclusions are based on an analytical and numerical study of the model chemical reaction formed when two diabatic harmonic potential-energy

25 surfaces are coupled linearly through a single vibration (sometimes called the Jahn-Teller $E \otimes B$ Hamiltonian or the two-site Holstein model). This model is solved over a large parameter space focusing on both the lowest-energy spectroscopic transitions and the quantum dynamics of coherent-state wavepackets. In addition, the properties of model chemical systems are considered including: ammonia inversion, aromaticity in benzene, the Creutz-Taube ion, the bacterial photosynthetic reaction centre,

${ }_{30} \mathrm{BNB}$, the molecular conductor Alq3, and inverted-region charge recombination in a ferrocene-porphyrinfullerene triad photosynthetic model compound. The range of the parameter space in which the BO approximation works well is also described. Throughout, the fundamental nature of BO breakdown is linked to the properties of the Cusp Catastrophe: the cusp diameter is shown to determine the magnitudes of all couplings, numerical basis-set and trajectory-integration requirements, and to simply depict the 35 transmission coefficient $\kappa$ often used to model deviations from transition-state theory.

\section{Introduction}

The Born-Oppenheimer (BO) adiabatic approximation ${ }^{1-4}$ was introduced in 1927 and provides a mathematical basis for the interpretation of basic chemical processes, processes whose 40 fundamental quantum nature were understood very soon after the introduction of quantum mechanics in $1925 .^{5-7}$ It delivers the concept of molecules as collections of distinguishable moving atoms, ${ }^{4}$ specifying molecular potential-energy surfaces constructed simply by solving the quantum motion of the 45 electrons repeatedly at different molecular geometries; significantly, such surfaces do not depend on the nuclear motion.
Analyses of these BO surfaces for small displacements from equilibrium geometries adequately describe much of chemical thermodynamics and spectroscopy, whilst transition-state theory, 50 in either its traditional classical Arrhenius form or its generalized quantum form including through-barrier tunneling, describes much of chemical reactivity. However, a range of thermodynamic, spectroscopic and kinetic phenomena require in addition explicit treatment of the vibronic coupling between 55 nuclear motion and electronic wavefunctions. Finding conceptually enlightening and/or quantitatively accurate solutions in such cases is at the forefront of research in chemical physics, ${ }^{8-}$ 14 involving identification and treatment of the causes for 
breakdown of the $\mathrm{BO}$ approximation

While it is possible to avoid the BO approximation by directly solving the coupled quantum electron-nuclear dynamics, ${ }^{15-20}$ the associated computationally difficulty leads to it being much more 5 common to start with the Born-Oppenheimer approximation, calculate potential energy surfaces, then take into account BO breakdown. Two fundamentally different strategies are in common use for the treatment of $\mathrm{BO}$ breakdown. The adiabatic approach starts with the $\mathrm{BO}$ approximation and in an a priori 10 fashion seeks to determine directly the consequences of its shortcomings on observable properties. ${ }^{11,21-34}$ It has the advantage of requiring in principle no approximations and therefore of being able to deliver the "exact" answer. In competition to this is the diabatic approach that transforms the 15 representation of the electronic states from the $\mathrm{BO}$ basis into diabatic states, also known as crude-adiabatic (CA) states, ${ }^{3}$ in which not all of the electronic interactions are represented in diagonal (eigenstate) form. This process has the advantage that it allows complex adiabatic surfaces to be represented in much 20 simpler form; ${ }^{35-39}$ for example, anharmonic reactive BO potentialenergy surfaces can be transformed to an essentially harmonic representation. In this process, complex problems involving chemical reactions can be described in simple, chemically intuitive ways to provide an excellent qualitative picture of the 25 critical processes involved. It has the disadvantage that no diabatic representation is unique, that nuclear exchange is not included, and the success of the method is directly correlated with the chemical intuition (entered either manually or else using some automated procedure) used in the construction of the 30 diabatization. ${ }^{11,19,21,26-28,34,40-55}$ Another advantage of the diabatic approach is that numerically converged full quantum solutions are typically easy to obtain whereas this process is often very difficult when using expansions in adiabatic basis functions. So it is usually quite easy to obtain a "good" answer via a diabatic

35 approach but difficult to obtain the "exact" answer; the process of diabatization, including especially ways of automating it, is thus of significant current interest. ${ }^{4,11-14,26,34,46,51,52,54,55}$ Electronic states obtained from a priori calculations using some physically motivated equation of constraint are usually termed quasidiabatic 40 states. $^{39,56}$

In this work we are concerned with the general applicability of the BO approximation and, more importantly, the fundamental nature, mathematical form, varying significance, and inherent interconnectivity of the terms that lead to its breakdown. It is

45 well known that, neglecting nuclear exchange, breakdown of the BO approximation stems from three neglected contributions to the molecular energy operator: the adiabatic diagonal correction (DC), the first-derivative (nuclear momentum) coupling (FD), and the second-derivative (nuclear kinetic-energy) coupling 50 (SD). ${ }^{2,3}, 57$ The diagonal correction modifies BO surfaces to produce mass-dependent Born-Huang adiabatic potential-energy surfaces, $3,45,57,58$ whilst the other two terms introduce nonadiabatic couplings between the BO states. 2, 3, 45, 57 While powerful general relationships relating the operators which 55 specify these effects are known, ${ }^{10,59-61}$ practical computational methods rarely exploit them but instead choose to focus on just the contribution believed to be dominant.

The DC modifies the shape of the potential-energy surfaces, changing heat of formation, vibrational frequencies, transition60 state energies, etc., and the application of this correction can naively be thought of as just improving the quality of $\mathrm{BO}$ surfaces without changing their fundamental character. ${ }^{62}$ However, the DC is mass dependent and so different sets of Born-Huang adiabatic potential-energy surfaces are produced for ${ }_{65}$ each molecular isotope, introducing mass dependence in a far more profound way than occurs say simply by the inclusion of tunneling corrections or zero-point energy effects for properties evaluated within the BO approximation. Diagonal correction is sometimes applied in calculations of infrared spectra, equilibrium 70 geometries, and chemical reaction energies, with results suggesting that its widespread adoption as a standard procedure is now warranted. ${ }^{3,57,63-91}$

The FD and SD corrections are non-adiabatic and so mix the potential-energy surfaces, eliminating after their application ${ }_{5}$ critically useful qualitative chemical descriptors such as the concept of molecular vibration frequencies and the concept of a transition state. Nevertheless, the FD correction is widely applied during the study of chemical reactions as this is the lowest-order correction that induces surface hopping. Conversely, the SD 80 correction is rarely applied because it is a higher-order correction $^{9,60,92}$ and FD alone often appears to suffice. ${ }^{19}$

Currently, the FD correction is evaluated by a range of quantum-chemistry software packages for applications to nonadiabatic processes. ${ }^{12}$ The SD correction is rarely evaluated ${ }_{85}$ but can be, ${ }^{67}$ while a small but steadily increasing number of software packages evaluate the DC term. ${ }^{66-74}$ Important tools for the evaluation of the DC and SD corrections have been developed, ${ }^{10,59-61}$ tools that imply a strong connection between the different corrections but yet remain underexploited in 90 practical applications. Recently, however, Kutznelnigg ${ }^{62}$ has shown that, for the model molecule $\mathrm{H}_{2}{ }^{+}$, the $\mathrm{DC}$ term is unexpectedly the dominant correction for all properties considered. Excellent modern discussions of these issues are contained in his work and elsewhere. ${ }^{52}$

95 So at the moment we see that a variety of effective computational methods focusing on different fundamental properties are available or developable to treat BO breakdown in a wide range of scenarios, but there is no general understanding as to how they fit together into a holistic picture. From a very 100 general mathematical perspective, BO breakdown is known to provide an example of a pitchfork-bifurcation cusp catastrophe. ${ }^{93-95}$ Such systems are by their very nature extremely sensitive to the details of their controlling parameters, are poorly suited to treatment through use of simplifying approximations, 105 and lead to numerical instabilities such as exponentially increasing errors with time during the numerical solution of dynamics near the cusp. In general, the naive ansatz that adding more of the correction terms simply produces a better answer in a monotonic way, as is typical of much of chemistry, would not be 10 expected to apply near a cusp. As a consequence, significant interplay between the DC, FD, and SD corrections is expected, and the standard chemical practice of considering only one of these terms in isolation is questioned.

Historically, the model systems used to consider the 115 consequences of BO breakdown for thermochemistry and vibrational spectroscopy have been fundamentally different to those used to describe photochemical reactions involving conical intersections. ${ }^{2}, 3,13,14,57,66-90,96,97$ For thermochemistry and vibrational spectroscopy, harmonic-oscillator models form the 20 basis of discussion, models that do not allow for chemical reactions. However, non-BO effects in ground-state chemical reactions are usually considered using methods developed for conical intersections, ${ }^{13,14,97,98}$ although specific approaches such as the "small polaron" model have proven useful. ${ }^{96}$ Further, it is 125 well known that the effects of BO breakdown manifest quite differently during electron-transfer reactions in the "normal" and "inverted" regimes, ${ }^{96}$ and we find that typical high-energy photochemical processes dominated by conical intersections are also fundamentally different. These three scenarios are sketched 130 in Fig. 1. For most thermally activated chemical reactions 


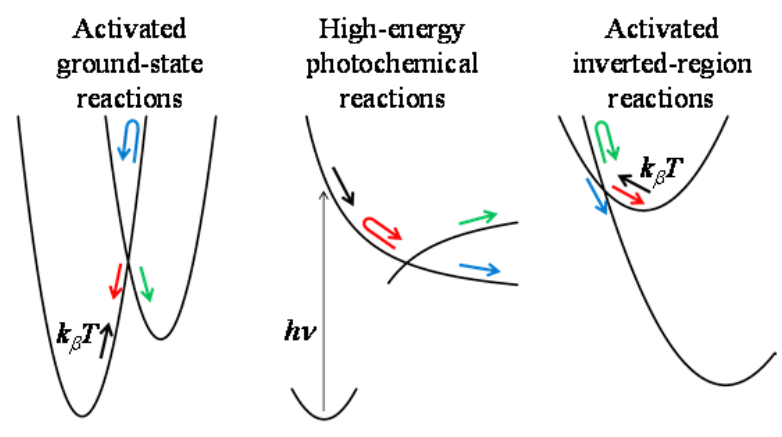

Fig. 1 Sketches of diabatic potential-energy surfaces and wavepacket dynamics for some classes of chemical reactions: (left) typical thermally activated chemical reactions, (centre) typical photochemical reactions in which the available energy exceeds that of relevant conical intersections, and (right) typical electron-transfer reactions in the inverted region that require thermal activation. Initial wavepackets (black) may be either directly transmit on the same BO surface (green), reflected back by the DC term (red), or forced to surface-hop by the FD or SD terms (blue).

including electron-transfer in the "normal" regime, a wavepacket incumbent on the transition state may either adiabatically cross the transition state to produce stable products or else be reflected back to regenerate stable reactants. In contrast, many photochemical reactions access conical intersection seams from an excited-state surface at an energy well above that of the conical intersection. ${ }^{81}$ Then reflection of the wavepacket merely leads to the original unstable species that must come back to reencounter the reactive region some time later, whilst passage

10 through this region can lead to two different stable products depending on whether surface hopping occurs or not. Reactions in the "inverted" region, however, usually involve thermal activation on the higher-energy surface but there is no transition state and so adiabatic passage and reflection both lead to no 15 reaction while surface hoping is required to produce products.

For high-energy reactions above that of the conical intersection, the inclusion of at least two vibrational motions is essential to properly include important Jahn-Teller and BObreakdown effects. However, only one nuclear coordinate is 20 required for a useful description of for many thermally-activated reactions (in both the "normal" and "inverted" regimes) that involve crossing from one potential-energy well to another along essentially a single nuclear coordinate, while general reactions of the form $\mathrm{A}+\mathrm{B} \rightarrow \mathrm{C}+\mathrm{D}$ in solution typically involves passing 25 through precursor and successor molecular complexes as so take on the form of an isomerization reaction $(\mathrm{AB}) \rightarrow(\mathrm{CD})$ during the critical stage. $^{92,99,100}$

Focusing on these latter situations which are well described by a single nuclear coordinate, we analytically and numerically 30 apply a chemical model to overview the effects of BO breakdown on ground-state chemical and spectroscopic properties, providing a unified description of thermochemistry, spectroscopy and lowenergy kinetics. This model is a two-diabatic-state Hamiltonian in which the two states are coupled by a single nuclear motion.

35 A very wide range of chemical processes can be described in terms of this simple qualitative model. Diabatic concepts underpin the perturbation theories of Landau and Zener from 1932 for infrequent processes in physics ${ }^{101-103}$ as well as London's more general treatment of non-adiabatic effects from 40 the same year. ${ }^{104}$ Eyring and Polanyi ${ }^{7}$ developed London's earlier ideas ${ }^{6}$ to construct the adiabatic LEP potential in 1931 and Horiuti and Polanyi in 1935 used them to describe proton and hydrogen transfer. ${ }^{105}$ This description was subsequently extended by Hush in 1953 to hydrogen-transfer oxidation-reduction 45 processes $^{99}$ and it now forms the basis of modern electrontransfer theory, ${ }^{106-110}$ describing for example exciton and charge transport through molecules, organic conductors and organic photovoltaics as well as electron-transfer reactions in biochemistry. $^{111-122}$ It also describes general racemization 50 processes. $^{123,124}$ Indeed, it has suggested that all chemical processes can be described in this way as a pseudo Jahn-Teller effect, $^{125,126}$ and we have shown ${ }^{100}$ that this model depicts the basic physics underlying the insightful reaction-force model ${ }^{127,128}$ of chemical reactivity. Further, the traditional language of 55 Herzberg-Teller theory ${ }^{125}, 129,130$ (a method widely applied in spectroscopic analyses $41,43,131$ ) can easily be recast into diabaticsurface concepts. Diabatic models are also of widespread interest in condensed-matter physics where this general approach is depicted in the Jahn-Teller $E \otimes B$ Hamiltonian ${ }^{132}$ and in the 60 two-site Holstein model. ${ }^{133}$

However, most of these chemical systems in reality display complex phenomena involving more than one type of nuclear motion and often involve more than two electronic states, limiting the quantitative applicability of our two-site one-mode model. ${ }_{65}$ Such complicating effects are not directly considered here but instead we consider purely the ability of calculations based on the BO approximation to accurately reproduce the easily calculable properties of the model system. If the BO approach is unable to reproduce the exact results for the model system, then it will also 70 perform poorly in more extensive treatments of the pertinent molecular systems.

Nevertheless, scenarios such as the Jahn-Teller effect, processes that occur at energies above that of a conical intersection, and reaction control by solvent friction do exist for 75 which a two-state single-model model is qualitatively inappropriate, and these are discussed in Electronic Supporting Information (ESI). 11, 50, 59, 97, 132, 134-139 Also, any problem involving closed-shell molecules in principle inherently involves more than two electronic states, but we make use of a 80 renormalization scheme ${ }^{140}$ that maps general problems of this nature onto an effective two-state model at the expense of making the physical meaning of the diabatic-surface parameters context sensitive. Useful applications of effective two-state models have been made even for reactions at metal surfaces for which an 85 infinite number of electronic states are involved. ${ }^{141}$

As the parameter space of the model is very large, we focus discussion using 10 molecular example systems and a further 8 characteristic sample points. The single-mode two-state model is in fact semi-quantitatively accurate for all of these 10 molecular 90 systems but the other sample points are selected more generally and do include regions that would be dominated by Jahn-Teller effects. $^{59,132}$ This extension is done so as to facilitate a more complete depiction of the properties of the model.

The 10 molecular systems considered are shown in Fig. 2: the 95 tunneling inversion motion in gas-phase ammonia, ${ }^{142}$ aromatic resonance in benzene, ${ }^{142}$ breakdown of aromaticity in the triplet $\left(\pi, \pi^{*}\right)$ ground-state of pyridine $\left({ }^{3} \mathrm{PYR}\right),{ }^{47,}{ }^{142}$ the Creutz-Taube ion in aqueous solution (CT), ${ }^{143}$ possibly including an orthomethoxy substituent on the pyrazine ring (CT-OMe), ${ }^{144} \mathrm{a}$ 100 variant of this in which pyrazine is replaced by a dipyridyl polyene (DPP), ${ }^{145,146}$ the bacterial photosynthetic reaction centre from Rhodobacter sphaeroides (PRC), ${ }^{49}{ }^{147}$ gas-phase BNB, ${ }^{148}$ pertinent to organic light-emitting diodes (OLED)s and artificial photovoltaics the fastest hole-transfer process in the $\beta$-phase 05 crystal of the molecular conductor mer-tris(8hydroxyquinolinato)aluminum(III) (Alq3) ${ }^{149}$, and a ferroceneporphyrin-fullerene model triad molecule $\left(\mathrm{FcPC}_{60}\right)$ that 


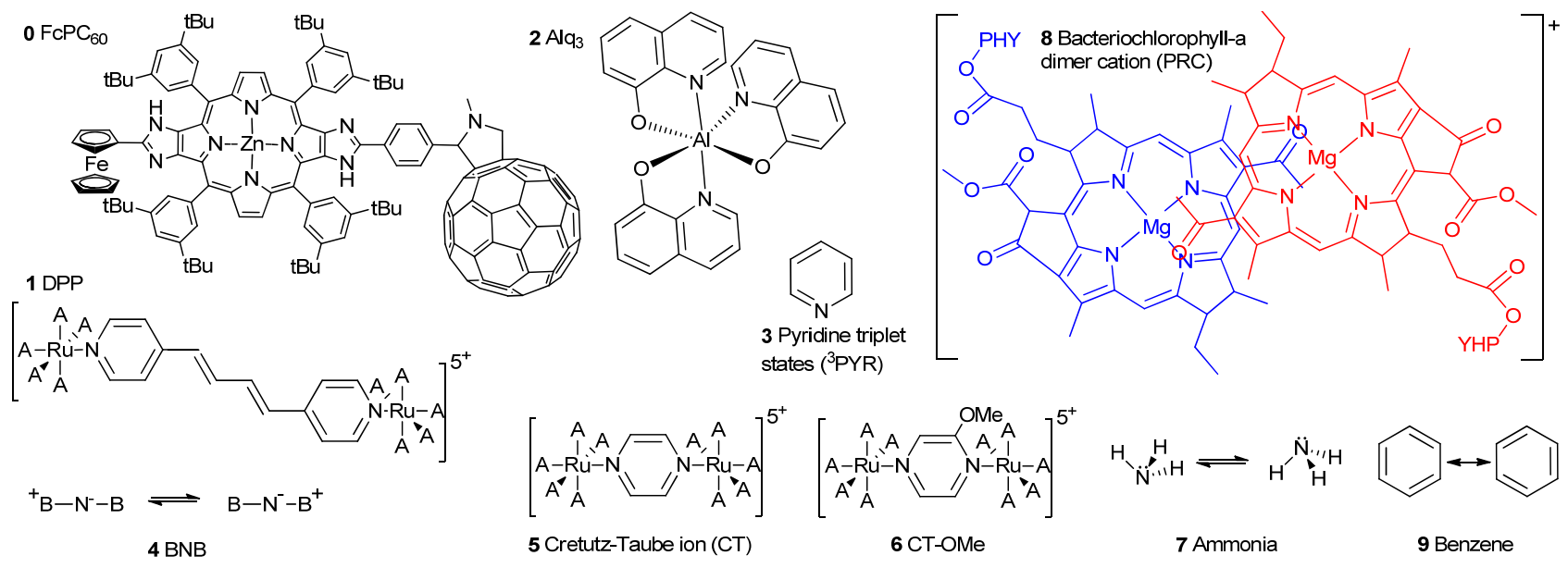

Fig. 2 Some sample molecular systems (see text) with electronic states that can be described using two coupled diabatic potential-energy surfaces. OMe is methoxy, PHY is phytyl; ${ }^{t} \mathrm{Bu}$ is tertiary butyl; A is ammonia; $\mathrm{FcPC}_{60}$ is Zinc, [[5,10,16,21-tetrakis[3,5-bis(1,1-dimethylethyl)phenyl]-13-[4(1',5'-dihydro-1'-methyl-2' $H$-[5,6]fullereno- $C_{60}-I_{h}$-[1,9-c]pyrrol-2'-yl)phenyl]-1,12-dihydro-23H,25H-diimidazo[4,5-b:4',5'-l]porphin-2-yl$\left.\kappa N^{23}, \kappa N^{24}, \kappa N^{25}, \kappa N^{26}\right]$ ferrocenato(2-)]-, (SP-4-1); Alq3 is mer-tris(8-hydroxyquinolinato)aluminum(III); DPP is Ruthenium(5+), decaammine[ $\mu$-[4,4'[(1E,3E)-1,3-butadiene-1,4-diyl]bis[pyridine- $\kappa N]]]$ di- (9CI).
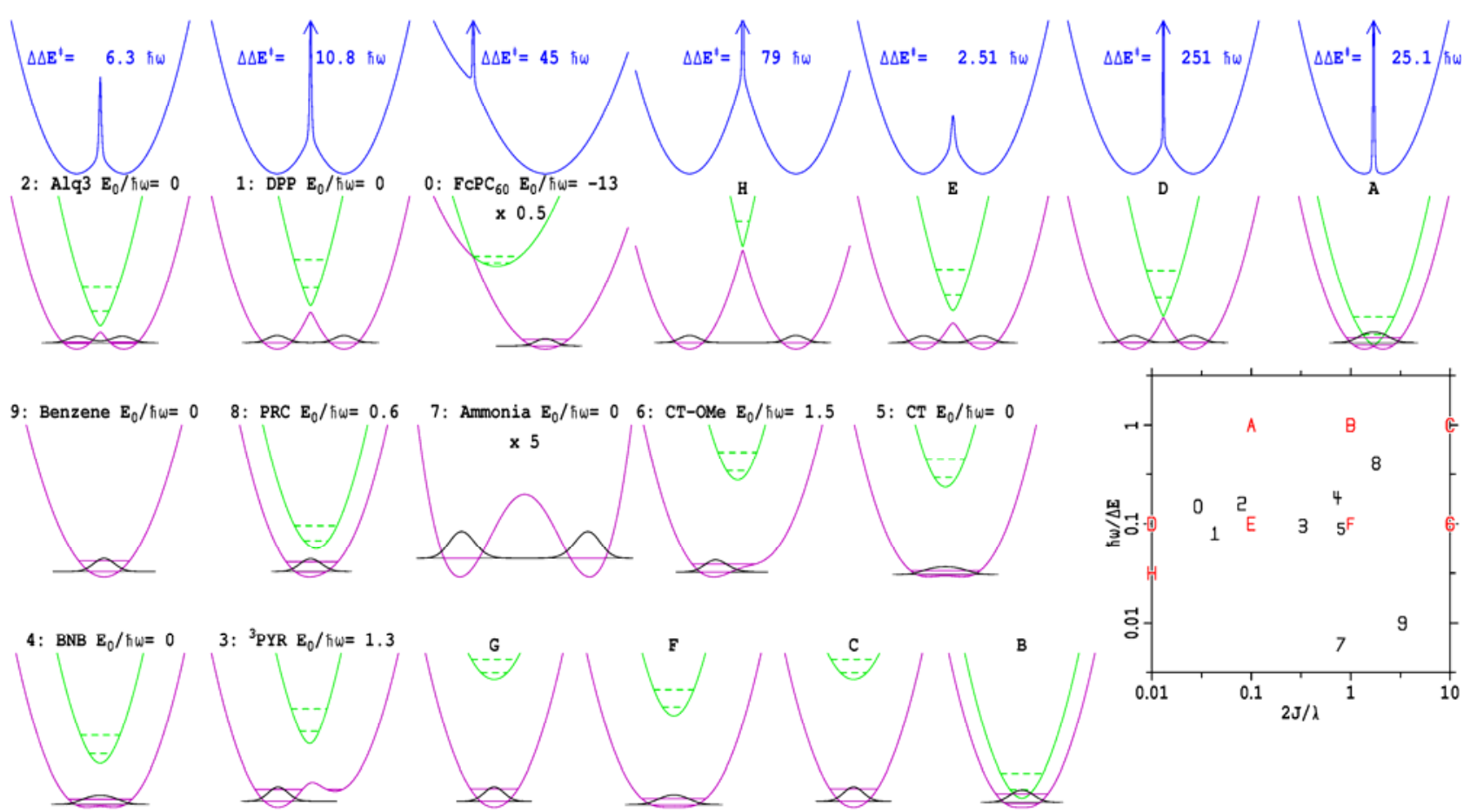

Fig. 3 Adiabatic potential-energy surfaces (relative energy $E / \hbar \omega$ vs. nuclear coordinate $Q$ ) at the characteristic points $\mathbf{A}-\mathbf{H}$ and for model compounds $\mathbf{0}-\mathbf{9}$ (see Table 1 and Fig. 2, 5 and $\mathbf{6}$ coincide on the projection shown), featuring (1) the BO ground-state (magenta) $\varepsilon_{-}(Q)$ and excited-state (green) $\varepsilon_{+}(Q)$ surfaces (Eqn. (3)) and their lowest two vibrational eigenstates, as well as the vibrational density of the lowest energy level in black, and (2) the Born-Huang adiabatic ground-state surface $\varepsilon_{-}^{B H}(Q)($ Eqn. $(10))$ in blue for the cases in which the change in activation energy $\Delta \Delta E^{\dagger}$ (Eqn. (23)) is larger than the original BO activation energy (Eqn. (21)).

undergoes long-range photochemical charge recombination. ${ }^{150,151}$ Limitations of the application of the one-mode model to these molecular systems are discussed in the ESI. ${ }^{49,150,152,153}$

For the model systems, the BO potential-energy surfaces 5 describing the chemical processes of interest are sketched in Fig. 3 and include double-well potentials with localized vibrations that are both strongly coupled (e.g. ammonia) and weakly coupled (e.g. Alq3, and DPP in the "normal" region and $\mathrm{FcPC}_{60}$ in the "inverted" region"), well-separated delocalized ground-state (GS) 10 and excited-state (ES) BO surfaces (e.g., benzene), double-well potentials that do not support zero-point motion (e.g., BNB), asymmetric systems which retain a double-well structure in which each well supports zero-point vibration (e.g., ${ }^{3} \mathrm{PYR}$ ), and asymmetric systems in which the delocalization is sufficient to 15 overcome the asymmetry (e.g., PRC). While study of the iconic Creutz-Taube ion paved the way for the modern understanding of 
strongly coupled molecular charge-transfer systems (e.g., as found in natural photosynthesis), its properties remain controversial, ${ }^{143}$ here it is depicted as a double-well system that does not support zero-point vibration; the molecule CT-OMe 5 interestingly introduces a slight asymmetry into the scenario. ${ }^{144}$

In Section 2 the model Hamiltonian and its solution using both efficient CA-based methods and often highly inefficient BObased methods is described, introducing explicit analytical expressions for the DC, FD, and SD corrections. Section 3 10 considers the physical meaning of the parameter space of the model, focusing on the properties of the 10 model systems as well as other characteristic points in the parameter space. Section 4 provides the main results, comparing the formally equivalent solutions obtained using CA-based methods with those obtained 15 using the BO approximation after the application of all 3 correction terms. Then these solutions are compared to ones obtained using either none or just one or else two of the BO correction terms. The analyzed properties include the nature of the lowest-two vibronic levels and the spectroscopic transition 20 between them, the nature of eigenstates near the transition state, the nature of spectroscopic transitions near the transition state, and the dynamics of coherent-state wavepackets depicting chemical reactions. Further, all results are analyzed in terms of the diameter of the cusp at the transition state and the degree of 25 technical difficulty required to actually solve for properties using a BO description. Finally, the results of the quantum wavepacket propagations are compared to those obtained from LandauZener ${ }^{102,} 103$ non-adiabatic reaction-rate theory and also its weakcoupling Golden-Rule approximation. ${ }^{107,154,155}$ Subsequently, 30 we look at how entanglement between the BO states can be used to quantify BO breakdown. ${ }^{156}$

\section{Methods}

The model Hamiltonian expresses the total electron-vibration molecular Hamiltonian $\mathbf{H}$ containing the electronic and nuclear 35 kinetic energies, the electron-electron, electron-nuclear and nuclear-nuclear Coulomb potential energies, and the electronelectron exchange energies (but neglects nuclear exchange) in terms of the diabatic states $\phi_{L}^{C A}\left(r, Q_{0}\right)$ and $\phi_{R}^{C A}\left(r, Q_{0}\right)$ as

$$
\begin{gathered}
\mathbf{H}^{C A}(Q)=\left[\begin{array}{ll}
H_{L L}^{C A}(Q) & H_{L R}^{C A}(Q) \\
H_{R L}^{C A}(Q) & H_{R R}^{C A}(Q)
\end{array}\right] \text { where } \\
H_{L L}^{C A}(Q)=\left\langle\phi_{L}^{C A}\left(r, Q_{0}\right)|\mathbf{H}| \phi_{L}^{C A}\left(r, Q_{0}\right)\right\rangle=\frac{\hbar \omega}{2}\left(Q+Q_{m}\right)^{2}-\frac{\hbar \omega}{2} \frac{d^{2}}{d Q^{2}} \\
H_{R R}^{C A}(Q)=\left\langle\phi_{R}^{C A}\left(r, Q_{0}\right)|\mathbf{H}| \phi_{R}^{C A}\left(r, Q_{0}\right)\right\rangle=\frac{\hbar \omega}{2}\left(Q-Q_{m}\right)^{2}-\frac{\hbar \omega}{2} \frac{d^{2}}{d Q^{2}} \\
+E_{0}
\end{gathered}
$$$$
H_{L R}^{C A}(Q)=\left\langle\phi_{L}^{C A}\left(r, Q_{0}\right)|\mathbf{H}| \phi_{R}^{C A}\left(r, Q_{0}\right)\right\rangle=J
$$$$
H_{R L}^{C A}(Q)=\left\langle\phi_{R}^{C A}\left(r, Q_{0}\right)|\mathbf{H}| \phi_{L}^{C A}\left(r, Q_{0}\right)\right\rangle=J
$$

and $J$ is the electronic coupling between the diabatic states, $r$ represents the electronic coordinates, $Q$ represents the chosen antisymmetric nuclear coordinate, $Q_{m}$ is a displacement that locates the two harmonic potentials at different nuclear 45 geometries, $\omega$ is the vibration frequency of the harmonic diabatic oscillators in the absence of coupling, and $E_{0}$ is an energy asymmetry that represents the free-energy change in a chemical reaction. While asymmetric molecules and reaction paths intrinsically involve structures with different fundamental
50 vibration frequencies, the free-energy change between the two species is typically the dominant factor and so frequency asymmetry is neglected in this simple model. However, the use of different frequencies would be essential in any calculation of the properties of dissociative reactions (see Fig. 1) in which this 55 model is applied. The nuclear displacement coordinate $Q$ can be related to mass-weighted normal coordinates $q$ by ${ }^{157} Q=q / q_{\text {zpt }}$ where $q_{z p t}$ is the zero-point vibrational length ${ }^{157} q_{z p t}=\sqrt{\hbar / \omega}$.

Numerically exact energy levels and wavefunctions of this Hamiltonian may be determined in a number of ways, each of ${ }^{60}$ which is very efficient in some region of the parameter space. ${ }^{41-44,}$ $50,110,158-160$ Here we adopt a single approach for all points considered that utilizes a harmonic-oscillator basis $\operatorname{set}^{161}\left|\chi_{i}(Q)\right\rangle$ centered at $Q=0$, including all functions with vibrational quanta of $0 \leq i<N$. The final (sparse) symmetric Hamiltonian matrix ${ }_{65}$ elements in the electron-vibration product basis set \{ $\left.\left.\left.\left|\phi_{L}^{C A}\left(r, Q_{0}\right\rangle\right| \chi_{i}(Q)\right\rangle,\left|\phi_{R}^{C A}\left(r, Q_{0}\right\rangle\right| \chi_{i}(Q)\right\rangle\right\}$ are then given by

$$
\begin{aligned}
H_{i j}^{L L} & =\left\langle\chi_{i}(Q)\left|\left\langle\phi_{L}^{C A}\left(r, Q_{0}\right)|\mathbf{H}| \phi_{L}^{C A}\left(r, Q_{0}\right)\right\rangle\right| \chi_{j}(Q)\right\rangle \\
& =H_{j i}^{L L}=-\hbar \omega Q_{m} \sqrt{\frac{i+1}{2}} \delta_{j, i+1}+\left(i+\frac{1}{2}\right) \hbar \omega \delta_{i, j} \\
H_{i j}^{R R} & =\left\langle\chi_{i}(Q)\left|\left\langle\phi_{R}^{C A}\left(r, Q_{0}\right)|\mathbf{H}| \phi_{R}^{C A}\left(r, Q_{0}\right)\right\rangle\right| \chi_{j}(Q)\right\rangle \\
& =H_{j i}^{R R}=\hbar \omega Q_{m} \sqrt{\frac{i+1}{2}} \delta_{j, i+1}+\left[E_{0}+\left(i+\frac{1}{2}\right) \hbar \omega\right] \delta_{i, j} \\
H_{i j}^{L R} & =\left\langle\chi_{i}(Q)\left|\left\langle\phi_{L}^{C A}\left(r, Q_{0}\right)|\mathbf{H}| \phi_{R}^{C A}\left(r, Q_{0}\right)\right\rangle\right| \chi_{j}(Q)\right\rangle \\
& =H_{i j}^{R L}=J \delta_{i, j}
\end{aligned}
$$

when $j \geq i{ }^{161}$ Our chosen approach is computationally efficient whenever the number of bound levels inside a well below a 70 transition state is small, with typically only a few vibrational basis functions required for convergence. However, as a matter of routine, we use a large number of 256 basis functions per state throughout most of the parameter space. However, when $\hbar \omega / \Delta E<0.005$ and $2|J| / \lambda<1$, the number of vibrational levels

75 below the transition state can be very large and so we increase this to 512 functions.

An interpretation of this model is that the BO approximation demands that the coupling $J$ is zero and that other values intrinsically indicate BO breakdown. ${ }^{160}$ This interpretation is not 80 consistent with the historical interpretation of the BornOppenheimer states as being those obtained by parametrically diagonalizing the electronic Hamiltonian as a function of nuclear coordinate, however, ${ }^{52}$ and is not adopted herein; indeed, we show shortly that the $\mathrm{BO}$ corrections become undefined at $J=0$, 85 making this the worst-possible situation for the BO approximation which then manifests derivative discontinues in the potential-energy surfaces, while from Eqn. (1) it is clearly that for $J=0$ it is in fact the CA basis states that specify the exact solution. Here we apply the BO approximation in its standard 90 form to Eqn. (1), parametrically diagonalizing the electronic states as a function of nuclear coordinate $Q$, leading to GS and ES adiabatic potential-energy surfaces $\varepsilon_{-}(Q)$ and $\varepsilon_{+}(Q)$, respectively, where

$$
\varepsilon_{ \pm}(Q)=\frac{E_{0}}{2}+\frac{\lambda}{4}+\frac{\hbar \omega}{2} Q^{2} \pm\left[\left(\frac{E_{0}}{2}-\hbar \omega Q_{m} Q\right)^{2}+4 J^{2}\right]^{1 / 2}
$$

95 with the reorganization energy defined as

$$
\lambda=2 \hbar \omega Q_{m}^{2}
$$


(which may also be represented as $\hbar \omega$ times the Huang-Rhys factor $2 Q_{m}{ }^{2}$ commonly used in spectroscopic interpretations). It is these surfaces that are sketched in Fig. 3 in magenta (GS) and green (ES). The associated eigenvectors can be used to produce 5 new $\mathrm{BO}$ electronic basis states

$$
\begin{aligned}
& \left|\phi_{-}(r, Q)\right\rangle=a(Q)\left|\phi_{L}^{C A}\left(r, Q_{0}\right)\right\rangle+b(Q)\left|\phi_{R}^{C A}\left(r, Q_{0}\right)\right\rangle \\
& \left|\phi_{+}(r, Q)\right\rangle=-b(Q)\left|\phi_{L}^{C A}\left(r, Q_{0}\right)\right\rangle+a(Q)\left|\phi_{R}^{C A}\left(r, Q_{0}\right)\right\rangle
\end{aligned}
$$

where

$$
\begin{aligned}
& a(Q)=\frac{J}{\left\{J^{2}+\left[\varepsilon_{-}-\hbar \omega\left(Q+Q_{m}\right)^{2} / 2\right]^{2}\right\}^{1 / 2}}, \text { and } \\
& b(Q)=\frac{\varepsilon_{-}-\hbar \omega\left(Q+Q_{m}\right)^{2} / 2}{\left\{J^{2}+\left[\varepsilon_{-}-\hbar \omega\left(Q+Q_{m}\right)^{2} / 2\right]^{2}\right\}^{1 / 2}}
\end{aligned}
$$

Application of the standard BO approximation then proceeds 10 in its usual modern way, ${ }^{2,57}$ evaluating all molecular properties from these potential-energy surfaces and electronic wavefunctions. However, using the $\mathrm{BO}$ electronic states in Eqn. (5) as a new basis set, the Hamiltonian Eqn. (1) may be rewritten without approximation ${ }^{2,3}$ as $\mathbf{H}^{B O}(Q)$ where

$$
\begin{aligned}
& H_{a b}^{B O}(Q)=\left\langle\phi_{a}^{B O}(r, Q)|\mathbf{H}| \phi_{b}^{B O}(r, Q)\right\rangle \text { for } a, b=+,- \text { and } \\
& H_{--}^{B O}(Q)=\varepsilon_{-}(Q)+\Delta H^{D C}(Q)-\frac{\hbar \omega}{2} \frac{\partial^{2}}{\partial Q^{2}} \\
& H_{++}^{B O}(Q)=\varepsilon_{+}(Q)+\Delta H^{D C}(Q)-\frac{\hbar \omega}{2} \frac{\partial^{2}}{\partial Q^{2}} \\
& H_{-+}^{B O}(Q)=-H_{+-}^{B O}(Q)=\Delta P^{F D}(Q) \frac{\partial}{\partial Q}+\Delta H^{S D}(Q)
\end{aligned}
$$

Here,

$$
\begin{aligned}
\Delta H^{D C}(Q)= & \frac{-\hbar \omega}{2}\left(a(Q) \frac{\partial^{2} a(Q)}{\partial Q^{2}}+b(Q) \frac{\partial^{2} b(Q)}{\partial Q^{2}}\right) \\
& =\frac{\hbar^{2} \omega^{2} \lambda J^{2}}{4\left[2 J^{2}+\hbar \omega \lambda\left(Q-Q_{\mathrm{x}}\right)^{2}\right]^{2}} \\
\Delta P^{F D}(Q)= & -\hbar \omega\left(-a(Q) \frac{\partial b(Q)}{\partial Q}+b(Q) \frac{\partial a(Q)}{\partial Q}\right) \\
& =\frac{-\hbar^{2} \omega^{2} Q_{m} J}{2 J^{2}+\hbar \omega \lambda\left(Q-Q_{\mathrm{x}}\right)^{2}} \text { and } \\
\Delta H^{S D}(Q)= & \frac{-\hbar \omega}{2}\left(-a(Q) \frac{\partial^{2} b(Q)}{\partial Q^{2}}+b(Q) \frac{\partial^{2} a(Q)}{\partial Q^{2}}\right) \\
& =\frac{\hbar^{3} \omega^{3} \lambda J Q_{m}\left(Q-Q_{\mathrm{x}}\right)}{\left[2 J^{2}+\hbar \omega \lambda\left(Q-Q_{\mathrm{x}}\right)^{2}\right]^{2}}
\end{aligned}
$$

provide the $\mathrm{DC}, \mathrm{FD}$, and $\mathrm{SD}$ corrections $\Delta H^{D C}$, $\Delta H^{F D}=\Delta P^{F D}(Q) \partial / \partial Q$, and $\Delta H^{S D}$, respectively, to the BO 20 approximation, with also

$$
Q_{\mathrm{x}}=\frac{E_{0}}{\lambda} Q_{m}
$$

being the nuclear coordinate at which the two CA states intersect. Note that as a result of the coordinate-dependent transformation,
$\mathbf{H}^{B O}(Q)$ appears antisymmetric in both the electronic and 25 nuclear coordinates but is symmetric overall; this feature generates the well-known selection rule that for symmetric molecules odd-quanta levels of one electronic state can only interact with even-quanta levels of the other.

An important feature is that the DC correction is always 30 positive, and in general the inclusion of this correction results in a variational method for which the calculated lowest vibronic energy level (i.e., that which defines the heat of formation) is strictly greater than it's exact energy. However, Eqn. (8) shows that all $\mathrm{BO}$ corrections become undefined ("0/0") at $Q=Q_{\mathrm{x}}$

35 when $J=0$ because of the manifested derivative discontinuity, making impossible the determination of the exact answer using an adiabatic approach; however, the CA basis states provide the analytical solution to the exact answer in this case. The BO adiabatic approximation is defined by Eqns. (3-6) and neglects all 40 three corrections in Eqn. (8), while numerically exact solutions to the original Hamiltonian Eqn. (1) can be obtained by including them all (we term this the "full calculation" $\mathrm{FC}=\mathrm{DC}+\mathrm{FD}+\mathrm{SD}$ ), with a variety of intermediary approximations also being possible such as the inclusion of just the DC correction to produce the ${ }_{45}$ Born-Huang adiabatic potential-energy surfaces, 37,59

$$
\varepsilon_{ \pm}^{B H}(Q)=\varepsilon_{ \pm}(Q)+\Delta H^{D C}(Q) .
$$

To evaluate vibrational matrix elements of the Hamiltonian in the BO electronic-state basis, we use the same centered harmonic-oscillator basis set as used previously to evaluate 50 matrix elements of the Hamiltonian in the crude-adiabatic basis as this is the most computationally efficient procedure. Required matrix elements such as $\left\langle\chi_{i}\left|\varepsilon_{-}(Q)\right| \chi_{j}\right\rangle$ are evaluated numerically while analytical integrals ${ }^{157}$ are used for the nuclear kinetic energy integrals and the effects of the momentum operator

$$
{ }_{55} \quad \frac{\partial}{\partial Q}\left|\chi_{j}(Q)\right\rangle=-\sqrt{\frac{j+1}{2}}\left|\chi_{j+1}(Q)\right\rangle+\sqrt{\frac{j}{2}}\left|\chi_{j-1}(Q)\right\rangle .
$$

Other vibrational basis sets such as plane waves offer advantages through say the use of Fourier-transform technology but typically need four times the number of basis functions to achieve a similar level of convergence as found for the harmonic-oscillator basis.

60 The numerical determination of the vibronic energy levels and eigenfunctions then proceeds simply by selecting the desired level of approximation $\left(\varepsilon_{ \pm}(Q)\right.$ plus one, two, or all of the BO correction terms $\Delta H^{D C}, \Delta H^{F D}=\Delta P^{F D} \partial / \partial Q$, and $\left.\Delta H^{S D}\right)$, constructing the integrals using a vibrational basis set truncated at ${ }_{65} N$ levels, and diagonalizing the resulting matrix to yield the $2 N$ (numerically exact) eigenfunctions

$$
\left|\psi_{j}(r, Q)\right\rangle=\sum_{+,-i=1}^{N} \sum_{i j}^{ \pm}\left|\phi_{ \pm}(r, Q)\right\rangle\left|\chi_{i}(Q)\right\rangle
$$

and associated energy levels $\varepsilon_{j}$. It is, however, conceptually convenient to consider the full Hamiltonian and the partially70 corrected Hamiltonians in the basis of the vibrational eigenfunctions

$$
\left|\phi_{j}^{\prime \pm}(r, Q)\right\rangle=\sum_{i=1}^{N} c_{i j}^{ \pm}\left|\phi_{ \pm}(r, Q)\right\rangle\left|\chi_{i}(Q)\right\rangle
$$

of the BO Hamiltonian. The BO Hamiltonian in this basis is simply a diagonal matrix with elements $\varepsilon_{i}^{ \pm}$, i.e.,

$$
\left\langle\varphi_{i}^{\prime \pm}(r, Q)\left|\varepsilon_{ \pm}(Q)\right| \phi_{j}^{\prime \pm}(r, Q)\right\rangle=\delta_{i j} \varepsilon_{i}^{ \pm},
$$

allowing specific inter-level effects of the BO breakdown terms to be explicitly manifested. 
Table 1 Estimates (see text and ESI) of parameters values for the coupled harmonic potential-energy surfaces for molecular systems $\mathbf{0}$ - 9 (see Fig. 2) and characteristic points $\mathbf{A}-\mathbf{H}$, along with the evaluated properties: $N$ - minimum number of BO vibrational basis functions per state needed to converge the lowest two vibronic levels to $0.2 \%$; $Q_{C}$ - the cusp diameter (Eqn. (17)), $\Delta \Delta E^{\dagger}=\left(\Delta H^{D C}\right)_{\max }$ is the DC contribution to the activation energy (Eqn. (23)); $\Delta \varepsilon_{0}$ - error in the energy of the lowest vibronic level (Eqn. (29)), and $\Delta v_{01}$ - error in the lowest transition energy (Eqn. (30)), evaluated using the BO approximation or this plus either the DC correction (Eqn. (10)), or the FD correction only; $\kappa$ - transmission coefficient from Landau-Zener (L-Z) theory (Eqns. (38-40), its Golden-Rule (G-R) approximation (Eqn. (48)) and crudely estimated from approximate coherent-state dynamics (DC, FD) compared to the exact dynamics (CA); see text for the sources of the molecular parameters

\begin{tabular}{|c|c|c|c|c|c|c|c|c|c|c|c|c|c|c|}
\hline System & $\frac{2|J|}{\lambda}$ & $\frac{\hbar \omega}{\Delta E}$ & $\frac{E_{0}}{\hbar \omega}$ & $N^{\mathrm{b}}$ & $Q_{C}$ & $\frac{\Delta \Delta E^{\dagger}}{\hbar \omega}$ & $\frac{\Delta \varepsilon_{0}^{B O}}{\hbar \omega}$ & $\frac{\Delta \varepsilon_{0}^{D C}}{\hbar \omega}$ & $\frac{\Delta v_{01}^{B O}}{\hbar \omega}$ & $\frac{\Delta v_{01}^{D C}}{\hbar \omega}$ & $\frac{\Delta v_{01}^{F D}}{\hbar \omega}$ & $\frac{\kappa^{G-R}}{\kappa^{L-Z}}$ & $\frac{\kappa^{D C}}{\kappa^{C A}}$ & $\frac{\kappa^{F D}}{\kappa^{C A}}$ \\
\hline 0 $\mathrm{FcPC}_{60}$ & 0.029 & 0.15 & -13 & 2 & 0.06 & 45 & 0.000 & 0.000 & 0.000 & 0.000 & 0.000 & - & - & - \\
\hline 1 DPP & 0.043 & 0.08 & 0 & 4 & 0.11 & 11 & -0.003 & 0.001 & 0.005 & 0.002 & 0.001 & 1.17 & 2.86 & 4.67 \\
\hline 2 Alq3 & 0.08 & 0.16 & 0 & 128 & 0.14 & 6.3 & -0.046 & 0.011 & 0.074 & 0.020 & 0.032 & 1.21 & 2.41 & 3.88 \\
\hline $3^{3} \mathrm{PYR}$ & 0.3 & 0.095 & $1.3^{\mathrm{a}}$ & 4 & 0.67 & 0.28 & -0.004 & 0.001 & -0.019 & 0.003 & -0.019 & 8.1 & 1.02 & 1.13 \\
\hline $4 \mathrm{BNB}$ & 0.74 & 0.18 & 0 & 4 & 1.11 & 0.10 & -0.045 & 0.005 & 0.043 & 0.013 & 0.035 & 14 & 1.03 & 1.07 \\
\hline $5 \mathrm{CT}$ & 0.80 & 0.089 & 0 & 2 & 1.68 & 0.045 & -0.026 & 0.001 & 0.016 & 0.003 & 0.014 & 46 & 0.96 & 0.99 \\
\hline 6 CT-OMe & 0.80 & 0.089 & $1.5^{\mathrm{a}}$ & 2 & 1.68 & 0.045 & -0.013 & 0.001 & -0.007 & 0.002 & -0.009 & - & - & - \\
\hline $7 \mathrm{NH}_{3}$ & 0.80 & 0.006 & 0 & 2 & 6.45 & 0.003 & -0.001 & 0.000 & 0.000 & 0.000 & 0.000 & 2650 & 0.88 & 0.88 \\
\hline 8 PRC & 1.8 & 0.41 & 0.6 & 4 & 1.39 & 0.065 & -0.029 & 0.009 & 0.036 & 0.026 & 0.017 & - & - & - \\
\hline 9 Benzene & 3.3 & 0.010 & 0 & 2 & 12.6 & 0.001 & -0.001 & 0.000 & 0.000 & 0.000 & 0.000 & - & - & - \\
\hline A & 0.1 & 1 & 0 & $>1024$ & 0.07 & 25 & & & & & & 1.02 & & \\
\hline B & 1 & 1 & 0 & 16 & 0.59 & 0.35 & -0.076 & 0.054 & 0.279 & 0.178 & 0.162 & - & - & - \\
\hline C & 10 & 1 & 0 & 4 & 2.23 & 0.025 & -0.011 & 0.010 & 0.114 & 0.109 & 0.022 & - & - & - \\
\hline D & 0.01 & 0.1 & 0 & 512 & 0.02 & 250 & -0.010 & 0.006 & 0.017 & 0.002 & 0.033 & 1.01 & 5.0 & 41 \\
\hline $\mathbf{E}$ & 0.1 & 0.1 & 0 & 8 & 0.22 & 2.5 & -0.010 & 0.001 & 0.014 & 0.006 & 0.011 & 1.67 & 1.62 & 2.18 \\
\hline $\mathbf{F}$ & 1 & 0.1 & 0 & 2 & 1.88 & 0.035 & -0.024 & 0.001 & 0.014 & 0.003 & 0.011 & - & - & - \\
\hline G & 10 & 0.1 & 0 & 2 & 7.05 & 0.003 & -0.002 & 0.000 & 0.001 & 0.001 & 0.000 & - & - & - \\
\hline H & 0.01 & 0.03162 & 0 & 2 & 0.04 & 79 & 0.000 & 0.000 & 0.000 & 0.000 & 0.000 & 1.04 & 4.7 & 12.1 \\
\hline
\end{tabular}

a indicated in the figures at the approximate location of $E_{0} / \hbar \omega=1.4$.

${ }^{\mathrm{b}}$ in excess of $\mathrm{N}$, a minimum of 256 basis functions are actually used in all calculations except 1024 functions are used for the dynamics of $\mathbf{2}$, A, and $\mathbf{D}$ but the results for $\mathbf{A}$ are not near convergence.

\section{The parameter space of the model}

As presented in Eqn. (1), the one-mode coupled two-state model contains four parameters, $J, \delta, \omega$, and $E_{0}$. However, as in practice most situations involve a variety of coupling motions, we choose 5 to express as many properties as possible in terms of the reorganization energy $\lambda$ as it is often the total value of the reorganization energy summed over all coupling modes that is the most critical parameter; in such a multi-mode interpretation, $\omega$ becomes the average vibration frequency of all the coupled 10 modes. As shown by Hush, ${ }^{162}$ for the symmetric problem $\left(E_{0}=0\right)$ the curvature of the BO GS surface at $Q=0$ is given by

$$
\frac{\partial^{2}}{\partial Q^{2}} \varepsilon_{-}(Q)=\hbar \omega\left(1-\frac{\lambda}{2|J|}\right)
$$

and so the GS has a double well whenever $\lambda>2|J|$. Hence we select $2|J| / \lambda$ as a critical variable depicting the chemical 15 properties of the parameter space. In the language of Catastrophe Theory, ${ }^{93-95}$ the BO Hamiltonian at $2|J| / \lambda=1$ and $E_{0}=0$ undergoes a pitchfork bifurcation that produces spontaneous symmetry breaking.

For a second critical variable depicting the model's parameter 20 space, we select the vibrational energy to electronic energy ratio $\hbar \omega / \Delta E$, where

$$
\Delta E=\left(\lambda^{2}+4 J^{2}\right)^{1 / 2}
$$

is the $\mathrm{BO}$ electronic energy gap at the diabatic state minima ( $Q= \pm Q_{m}$ ) in the absence of asymmetry $E_{0}$ (nb., at the adiabatic 25 minimum-energy geometry the corresponding vertical transition energy is just $\lambda){ }^{140}$ While the derivation of the BO approximation was originally made based on an expansion in the ratio of the reduced nuclear mass of a diatomic molecule to the electron mass, ${ }^{1,4}$ the essential physical insight behind this 30 approximation is that it is possible to ignore the effects of the nuclear momentum and kinetic-energy operators on the electronic wavefunctions when the timescales (or energy scales) of the two types of motion are quite different. The ratio $\hbar \omega / \Delta E$ captures this physical insight in a simple and general way: lower values of 35 this parameter $\ll 1$ will always increase the accuracy of the BO approximation. The most significant way in which the massdependent effects of $\mathrm{BO}$ breakdown are manifest in this work is through the mass dependence of this ratio which stems from the mass dependence of the diabatic-state vibration frequency $\omega$. ${ }_{40}$ Finally, we scale the energy asymmetry by the vibration frequency to obtain a third parameter, $E_{0} / \hbar \omega$. In this way the actual value of the vibration frequency can be considered to be an arbitrary scale parameter. Its value is conserved throughout the various potential-energy surfaces shown in Fig. 3.

45 Indeed, Fig. 3 shows the BO potential-energy surfaces and the two lowest BO vibronic energy levels $\varepsilon_{0}^{ \pm}$and $\varepsilon_{1}^{ \pm}$for both the GS (-) and ES (+) surfaces, as well as the exact vibrational density ${ }^{142,163}$ of the lowest-energy vibronic level for the 10 sample molecules $\mathbf{0}$ - 9 and 8 characteristic points $\mathbf{A}-\mathbf{H}$, as 
detailed in Table 1. Also indicated in the figure are the locations of these systems on a 2-dimensional log-scale plot depicting $\hbar \omega / \Delta E$ and $2|J| / \lambda$. These plots consider the ranges $0.01 \leq 2|J| / \lambda \leq 10$ and $0.00316 \leq \hbar \omega / \Delta E \leq 3.16$, values 5 extreme enough so that the asymptotic limits of the parameter space are exposed. Values for ammonia and benzene are taken from our previous interpretation of spectroscopic data, ${ }^{140,}, 142,164$ along with results for $\mathrm{CT},{ }^{143}{ }^{3} \mathrm{PYR},{ }^{70,74}$ and PRC, ${ }^{49,}{ }^{147}$ while recent spectroscopic analyses are used for $\mathrm{BNB}^{148}$ and $\mathrm{FcPC}_{60} .{ }^{150}$ ${ }_{0}{ }^{151}$ Data for Alq3 are taken from a recent study of the most prolific hole-transport pathway through the $\beta$-phase of the Alq3 crystal, ${ }^{165} J$ and $\lambda$ for DPP are taken from our experimental spectroscopic interpretation ${ }^{145}, 146$ with the deduced typical solvent frequency for CT also applied, ${ }^{143}$ and CT-OMe is taken to 15 be $\mathrm{CT}$ perturbed by $E_{0}=0.15 \mathrm{eV} .^{144}$ Issues concerning the application of a one-model model to some of these molecules is described in the ESI. ${ }^{49,150,152,153}$

The sample molecules present parameters with the ranges $0.03 \leq 2|J| / \lambda \leq 3.3$ and $0.006 \leq \hbar \omega / \Delta E \leq 0.41$ only, with 6

20 systems being symmetric $(\Delta E=0)$ and for the remainder either $\Delta E / \hbar \omega=-13,0.6,1.3$, or 1.5 ; values for these later two systems are subsequently shown on figures obtained at the intermediary value of $\Delta E / \hbar \omega=1.4$ for brevity. Charge-transfer systems of importance can display a wide range of $2|J| / \lambda$ values, with for

25 example in Alq3 crystal the depicted process has $2|J| / \lambda=0.08$ but this is pertinent to the most prolific hole-transfer pathway, with other hole-transfer pathways having values orders of magnitude smaller while for electron-transfer in Alq3 crystal, $2|J| / \lambda$ can approach unity. ${ }^{165}$ However, it is uncommon to find

30 functional molecular systems with values of $\hbar \omega / \Delta E$ exceeding 0.5 as this requires high frequencies, weak coupling, and low reorganization energies. Nevertheless, the region with $\hbar \omega / \Delta E \approx 1$ depicting say a double-well molecule in which the well depth is too shallow to support zero-point vibration can be

35 important, whilst $\hbar \omega / \Delta E \gg 1$ could become manifest at e.g., conical intersections. As mentioned earlier, such conical intersections should be described using an enhanced model containing at least one symmetric vibration, but the results obtained for our simpler one-mode problem remain informative.

40 As the sample molecules do not probe this region, the iconic systems $\mathbf{A}-\mathbf{H}$ provide a more systematic coverage of the model's parameter space. Systems $\mathbf{A}-\mathbf{C}$ with $\hbar \omega / \Delta E=1$ depict different scenarios applicable when the vibrational and electronic energy spacings are equivalent, $\mathbf{D}, \mathbf{E}$ and $\mathbf{H}$ represent

45 the isomerization processes or electron transfer in a typical molecule with many bound vibrational levels and a well defined transition state separating two different isomers, $\mathbf{G}$ depicts aromatic molecules with delocalized electronic states, and $\mathbf{F}$ is an intermediate case with the ground-state BO frequency highly

so depressed with quartic contributions dominating the ground-state potential-energy surface.

\section{Results}

a. The cusp diameter, Born-Huang adiabatic potential-energy surfaces, and the fundamental relationships between the three ${ }_{55}$ Born-Oppenheimer breakdown contributions

The curvatures of the transition states shown in Fig. 3 for $\mathbf{A}, \mathbf{D}$ $\mathbf{E}, \mathbf{H}, \mathbf{0}\left(\mathrm{FcPC}_{60}\right), \mathbf{1}$ (DPP) and $\mathbf{2}$ (Alq3) are all very high, indicating the presence of a cusp through which the $\mathrm{BO}$ surfaces
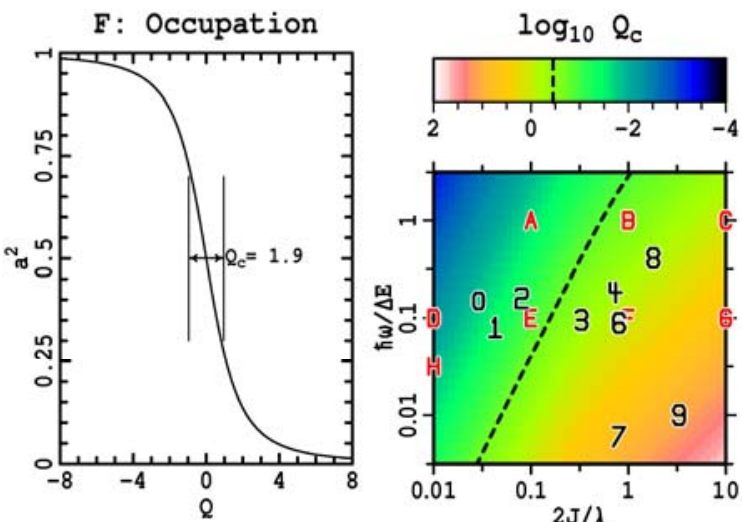

Fig. 4 Left: the change in the L-side occupation for scenario $\mathbf{F}$ (Eqn. (6)), illustrating the cusp diameter $Q_{c}$; Right: its variation over the parameter space from Eqn. (17). The dashed line shows where $Q_{c}=0.35$; from Eqn. (19) DC significantly modifies the transition-

state energy in the region to the left of this line and is therefore essential to include in kinetics calculations. Sample model compounds 0-9 and points A-H are indicated, see Table 1.

change rapidly from L-dominated (large $|a|$, small $|b|$ in Eqn. 6) to ${ }_{60}$ R-dominated (small $|a|$, large $|b|$ ); this change is illustrated in Fig. 4 for scenario $\mathbf{F}$. We define the cusp diameter as the distance between the two roots of $\partial^{3} a^{2}(Q) / \partial Q^{3}=0$ (see Eqn. (6)) i.e.,

$$
Q_{c}=\frac{2|J|}{\lambda} Q_{m}=\frac{2|J|}{\sqrt{2 \hbar \omega \lambda}} \approx 0.16\left(\frac{2|J|}{\lambda}\right)^{3 / 4}\left(\frac{\hbar \omega}{\Delta E}\right)^{-1 / 2} .
$$

This diameter is a characteristic descriptor of non-adiabatic ${ }_{65}$ processes and is shown in Fig. 4 as a function of the parameterspace investigated in this study wherein it varies by over 5 orders of magnitude. As the displacements $Q_{m}$ is in units of the length of the zero-point motion of the diabatic oscillators, the cusp diameter is mass dependent, a feature that is clearly apparent 70 from Fig. 4 via the shown dependence on $\hbar \omega / \Delta E$. Further, the approximate description of $Q_{C}$ given in Eqn. (17) (which is accurate to $\pm 40 \%$ over this and a wider parameter rang) indicates the expected ${ }^{1-4}$ (electron mass / nuclear mass) $)^{1 / 4}$ dependence for the breakdown of the BO approximation whilst simultaneously 75 quantifying the additional dependence of $\mathrm{BO}$ breakdown on the chemical property $2|J| / \lambda$

London's 1932 theory for non-adiabatic chemical reactions was the first to focus on the rapid change in $a^{2}(Q)$ that occurs as the cusp is crossed. ${ }^{104}$ Hush capitalized on this in his 1950's 80 adiabatic-based general theory of electron transfer, highlighting the partial charge transfer at the transition state, which allows transition-state properties to be tuned chemically, and the importance of the cusp and its properties to the breakdown of the BO breakdown. ${ }^{107,166-169}$ The cusp diameter is independent of 85 energy asymmetry $E_{0}$ and is appropriate independent of whether or not the ground-state adiabatic surface has a single well or a double well. Note that single wells arise owing to either delocalization or to large values of $\left|E_{0}\right|$ as would be found in the inverted region of chemical reaction; they occur whenever

$$
\left(\frac{E_{0}}{\Delta E}\right)^{2} \geq\left[1-\left(\frac{2|J|}{\lambda}\right)^{2 / 3}\right]^{3}\left[1+\left(\frac{2 J}{\lambda}\right)^{2}\right] .
$$

This relation implies a profound connection between the forces that lead to aromaticity and those that stabilize high-energy 
excited states in e.g. organic conductors and molecular photovoltaics. However, $Q_{C}$ will be large and hence BObreakdown small when resonance leads to a single well whereas it will be small and hence BO breakdown large in the inverted 5 region.

From Eqn. (8), the large values attained by the BO-correction terms in the region of the cusp are related to each other by

$$
\begin{aligned}
\Delta H^{B H}(Q) & =\frac{1}{2 \hbar \omega}\left(\Delta P^{F D}(Q)\right)^{2} \text { and } \\
\Delta H^{S D}(Q) & =2 \frac{\partial \Delta P^{F D}(Q)}{\partial Q}=\frac{2}{Q_{c} \hbar \omega}\left(Q-Q_{\mathrm{x}}\right)\left(\Delta P^{F D}(Q)\right)^{2} \\
& =\frac{4}{Q_{C}}\left(Q-Q_{\mathrm{x}}\right) \Delta H^{B H}(Q) .
\end{aligned}
$$

These equations provide specific examples of generally known 10 relationships ${ }^{9,60}$ that, independent of the number of vibrational modes or electronic states included, link the three BO-breakdown correction terms. For our model system, the maximum absolute values of $\Delta H^{D C}(Q)$ and $\Delta P^{F D}(Q)$ occur at $Q=Q_{\mathrm{x}}$, the point at which the $\mathrm{BO}$ states are equal mixtures of the $\mathrm{L}$ and $\mathrm{R} \mathrm{CA}$ 15 states, while the maximum value of $\Delta H^{S D}(Q)$ occurs at $Q=Q_{\mathrm{X}}+3^{-1 / 2} Q_{C}$; these maxima are:

$$
\begin{aligned}
& \left(\left|\Delta P^{F D}(Q)\right|\right)_{\max }=\frac{\hbar \omega}{2 Q_{C}}, \\
& \left(\Delta H^{D C}(Q)\right)_{\max }=\frac{\hbar \omega}{8 Q_{c}^{2}}, \text { and } \\
& \left(\Delta H^{S D}(Q)\right)_{\text {max }}=\frac{3 \sqrt{3} \hbar \omega}{32 Q_{C}^{2}}=\frac{3 \sqrt{3}}{4}\left(\Delta H^{B H}\right)_{\text {max }}
\end{aligned}
$$

and scale simply with the cusp diameter in the anticipated ${ }^{92}$ fashion. Hence this diameter is the critical physical property that 20 leads to BO breakdown. These equations show that the DC and SD corrections scale as the square of the FD correction and are thus higher order in a parameter that could be expected to be small if the effects of BO breakdown are weak. ${ }^{9,60,92}$ Note that while these equations suggest that the DC and SD contributions 25 should be equally important, the DC contribution appears directly as a diagonal contribution whereas the SD contributions must be weighted by the inverse energy gap between the ES and the GS and so is most competitive only near conical intersections; this effect also diminishes the impact of the FD correction. The effect 30 of the DC correction is to add a sharply spiked peak equally to both the GS and ES BO potential-energy surfaces, producing the Born-Huang adiabatic potential-energy surfaces specified in Eqn. (10). For systems $\mathbf{A}, \mathbf{C}, \mathbf{E}$, and $\mathbf{H}$ as well as for molecules $\mathbf{0}$ $\left(\mathrm{FcPC}_{60}\right), \mathbf{1}$ (DPP) and $\mathbf{2}$ (Alq3), the resulting GS surfaces ${ }_{35} \varepsilon_{-}^{B H}(Q)$ are compared in Fig. 3 to the original BO surfaces. The DC adds a sharp spike to the potential of height $\hbar \omega / 8 Q_{C}^{2}$ (Eqn. (20)) at or near the transition state, increasing the barrier for the chemical reaction by essentially this amount, $\Delta \Delta E^{\dagger}$. For symmetric reactions $\left(E_{0}=0\right)$ this increases the activation energy 40 from its $\mathrm{BO}$ value of ${ }^{140}$

$$
\Delta E^{\dagger}=\frac{\lambda}{4}\left(1-\frac{2|J|}{\lambda}\right)^{2}
$$

to that for the Born-Huang surface of

$$
\Delta E^{\dagger} \approx \frac{\lambda}{4}\left(1-\frac{2|J|}{\lambda}\right)^{2}+\frac{\hbar^{2} \omega^{2}}{8 J}\left(\frac{2|J|}{\lambda}\right)^{-1}
$$

which in the weak charge-transfer limit of $2|J| / \lambda \ll 1$ for ${ }_{45}$ arbitrary ${ }^{107} E_{0}$ is increased from its usual value in adiabatic electron-transfer theory ${ }^{107,154}$ by $\Delta \Delta E^{\dagger}$ :

$$
\begin{aligned}
& \Delta E^{\dagger} \approx \frac{\left(E_{0}+\lambda\right)^{2}}{4 \lambda}-|J|+\Delta \Delta E^{\dagger} \text { where } \\
& \Delta \Delta E^{\dagger}=\frac{(\hbar \omega)^{2} \lambda}{16 J^{2}}=\frac{\hbar \omega}{4}\left(\frac{\hbar \omega}{\lambda}\right)\left(\frac{2 J}{\lambda}\right)^{-2} .
\end{aligned}
$$

As a rough guide, the DC change to the transition-state energy will be significant whenever $\Delta \Delta E^{\dagger} / \hbar \omega>1$ or alternatively ${ }_{50} Q_{C}=Q_{m} \times 2|J| / \lambda<8^{-1 / 2}=0.35$, and the locus of this line is shown on Fig. 4 while cusp diameters calculated for the sample systems and molecules are given in Table 1. Catalysis at metal surfaces has been noticed to often be associated with chemical reaction barriers that are much larger than those predicted using ${ }_{55} \mathrm{BO}$ theory, and this field may provide key applications of this effect, ${ }^{141}$ but for high-energy photochemical processes, $\Delta \Delta E^{\dagger}$ would need to exceed the (possibly quite large) available excess energy above the transition-state for there to be an appreciable effect. Pertinent to thermal molecular processes, large activation60 energy changes occur for charge-transfer applications to molecules like $\mathbf{1}$ (DPP) and 2 (Alq3) for which $\Delta \Delta E^{\dagger} / \hbar \omega=10.8$ and 6.3, respectively, much larger than the original $\mathrm{BO}$ activation energies of $\Delta E^{\dagger} / \hbar \omega=2.8$ and 1.3 , respectively. Hence the DC terms cannot be neglected, contrary to standard practice.

65 Finally, we note that while the very sharply spiked nature of the BO-breakdown corrections is well known, only $\Delta P^{F D}(Q)$ is usually depicted graphically. ${ }^{11,32,34,170}$ This, however, is a momentum term that cannot be directly compared to energies and it is much more revealing to plot the Born-Huang adiabatic 70 potential-energy surfaces, as done in Fig 3.

\section{b. Convergence issues: The number of BO vibrational basis functions required to accurately determine properties of the full Hamiltonian.}

For situations in which the $\mathrm{BO}$ approximation is inadequate, 75 evaluation of the effects of BO breakdown using a BO rather than CA description is only feasible if the associated spectroscopic or quantum dynamics calculations can be converged using a realistic number of basis functions. In this subsection we focus on spectroscopic problems as the quantities involved are clearly 80 defined, determining the number of $\mathrm{BO}$ vibrational basis functions $\left|\phi_{j}^{\prime \pm}(r, Q)\right\rangle$ (Eqn. (13)) that must be included in order to obtain convergence of the calculated energies of the lowest two vibronic levels of the full Hamiltonian in the BO basis, Eqn. (7), to those simply obtained using Eqn. (1). We set the convergence 85 criterion to deliver an accuracy of $0.2 \%$ of $\hbar \omega$ for both levels. To converge quantum dynamics near a transition state, more basis functions are required, however, in proportion to the depth of the double well, see later.

In Table 1 and Fig. 5 is indicated the number $N$ of required BO ${ }_{90}$ basis functions per state (constrained to the form $N=2^{n}$ where $n$ is an integer) required to converge the lowest two vibronic levels. As it is possible that both of the $\mathrm{BO}$ vibronic eigenfunctions used in this process stem from the GS BO surface, the minimum possible value is $N=2$. We do not perform calculations above 


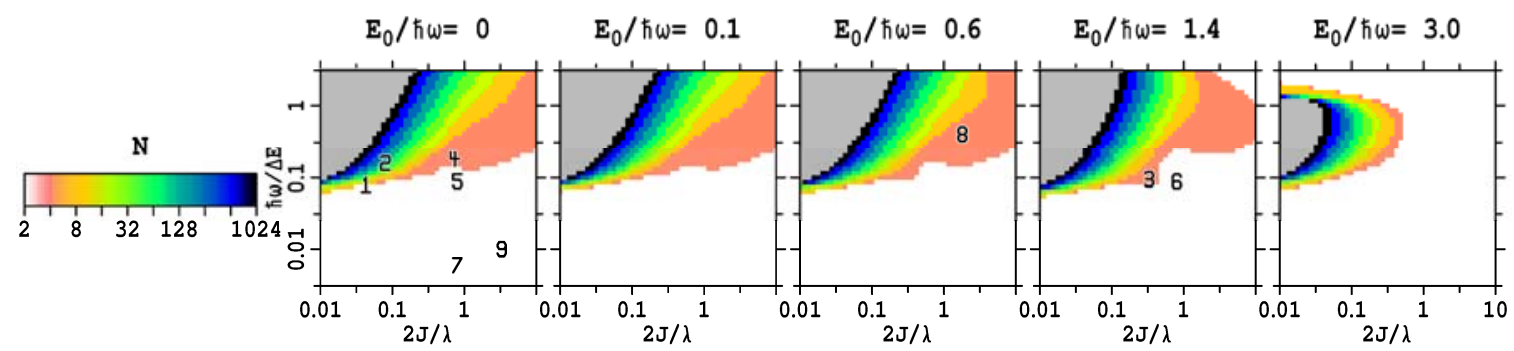

Fig. 5 The number $N$ of BO vibrational basis functions per state required for convergence of the lowest two energy levels to $0.2 \%$ ( $>1024$ in light gray regions). Sample model compounds 1-9 are indicated, see Table 1.

$N=1024$, instead graying-out in Fig. 5 (and later figures) the region of the parameter space for which $N=1024$ levels is inadequate.

For typical deep-welled molecular systems with $\hbar \omega / \Delta E<0.1$ 5 , the BO approximation works very well requiring the minimum value of just $N=2$ vibrational levels per BO electronic state. This indicates that either the $\mathrm{BO}$ approximation accurately reproduces the exact properties or else simple analytical methods like perturbation theory could be introduced to describe any $\mathrm{BO}$ 10 breakdown. However, the number of required functions increases steeply outside of this region as $\hbar \omega / \Delta E$ increases and $2|J| / \lambda$ falls, quickly exceeding our practical limit of $N=1024$. Of particular significance are the small changes in the parameters found capable of transforming the BO approximation from an 15 excellent description to a very poor one in the region of $2|J| / \lambda<0.1, \hbar \omega / \Delta E \approx 0.1$, a region of significant interest for charge (and exciton) transfer.

The poor convergence of calculations performed using a BO electronic basis via Eqn. (7) can be understood considering the ${ }_{20}$ cusp diameter $Q_{c}$, Eqn. (17). The DC, FD, and SD terms cause low-energy BO GS wavefunctions to mix with either GS or ES wavefunctions that have nodal spacings of the order of $Q_{c}$. Hence the number of basis functions required to achieve a specified level of convergence of molecular properties naively must ${ }_{25}$ increase in proportion to $1 / Q_{C}^{2}$. Indeed, this effect gives rise to the extreme sensitivity of $N$ evidenced in Fig. 5. Comparing the utilized value of the vibrational basis set size $N$ to $Q_{c}$, we find empirically for symmetric situations with $E_{0} / \hbar \omega=0$ that, for $\hbar \omega / \Delta E>0.1$,

$$
\begin{aligned}
& N \approx \max \left(2,6 Q_{C}^{-2}\right) \text { with } \\
& 6 Q_{C}^{-2}=\frac{12 \hbar \omega}{\lambda}\left(\frac{\lambda}{2 J}\right)^{2} \approx 40\left(\frac{2|J|}{\lambda}\right)^{-3 / 2} \frac{\hbar \omega}{\Delta E},
\end{aligned}
$$

although in a few percent of cases the numerically optimized value is significantly smaller indicating that the convergence conditions used are not always strict enough. From within our sampled parameter space, this expression predicts that the

35 maximum number of $\mathrm{BO}$ vibrational eigenfunctions per state that must be included in an accurate numerical solution of the full Hamiltonian occurs for the point $2 J / \lambda=0.01, \hbar \omega / \Delta E=3.16$ and is $N=380,000$.

Asymmetry mixes the ES into the GS, significantly influencing 40 the lowest two vibronic wavefunctions. For $E_{0}>0$, the BO GS skews towards the $\mathrm{L}$ diabatic minimum, taking the wavefunctions away from the transition-state region in which the $\mathrm{BO}$ breakdown is large. Hence Fig. 5 shows that the $\mathrm{BO}$ approximation performs much better for the low-energy levels of asymmetric potentials 45 than it does for symmetric ones, with the number of basis functions required for convergence decreasing significantly as the magnitude of $E_{0}$ increases. The most difficult region becomes that for double-well potentials $(2|J| / \lambda<1)$ with comparable vibrational and electronic energy gaps $(\hbar \omega / \Delta E \approx 1)$. This result ${ }_{50}$ has been previously noted ${ }^{148}$ and is associated with entanglement between electronic and vibrational motion. ${ }^{142}$

While Fig. 5 shown the minimum number of vibrational basis functions per electronic state needed for convergence of the lowest two levels to $0.2 \%$, all subsequently reported results were 55 performed using a minimum of 256 levels per state, ensuring convergence to much better than 1 in $10^{6}$ for most data points.

c. Shortcomings of calculations for the thermochemical energy of the lowest vibronic level and for the lowest spectroscopic transition energy evaluated using approximate ${ }_{60}$ BO-based methods.

The top row of Fig. 6 shows the error

$$
\Delta \varepsilon_{0}^{B O}=\varepsilon_{0}^{B O}-\varepsilon_{0}^{F C}
$$

in the lowest-energy level evaluated using the BO approximation whilst the third row shows the associated absolute error in the ${ }_{65}$ calculated spectroscopic first transition energy

$$
\left|\Delta v_{01}^{B O}\right|=\left|\left(\varepsilon_{1}^{B O}-\varepsilon_{0}^{B O}\right)-\left(\varepsilon_{1}^{F C}-\varepsilon_{0}^{F C}\right)\right| .
$$

Here FC stands for the full calculation using all three BO correction terms $\Delta H^{D C}, \Delta H^{F D}$, and $\Delta H^{S D}$ in Eqn. (7) (or equivalently using Eqn. (1) and the $\mathrm{CA}$ basis), and the $\mathrm{BO}$ 70 approximation is enforced by simply neglecting all three corrections. Note that while the lowest vibronic level always resides on the GS, the higher-energy level involved in the lowestenergy transition may originate from either the GS or the ES surfaces, and the transition energy may represent tunneling, a GS 75 vibrational transition, or a vibronic transition to the ES surface. For symmetric systems with $E_{0} / \hbar \omega=0$, the BO energy of the lowest vibronic level is found to always be less than the exact energy level but the difference is mostly small, the largest calculated error being $-0.2 \hbar \omega$ at $2 J / \lambda<0.01, \hbar \omega / \Delta E=1$. 80 The associated errors in the lowest transition energy adopt a similar pattern but the quantity is always overestimated and the magnitude of the error is much larger, up to $0.6 \hbar \omega$. Hence it is clear that the errors made by the $\mathrm{BO}$ approximation for the first excited vibronic level are always larger in magnitude to those 85 made for the ground state. As these quantities are evaluated using only the $\mathrm{BO}$ surfaces $\varepsilon_{ \pm}(Q)$ assuming that the sharply peaked cusp-derived quantities $\Delta H^{D C}, \Delta H^{F D}$, and $\Delta H^{S D}$ can be neglected, calculations at the $\mathrm{BO}$ level are rapidly convergent over the whole parameter space considered. The errors are a 90 maximum when the electronic and vibrational energy spacings are equivalent $(\hbar \omega / \Delta E=1)$ and the GS surface has a double 


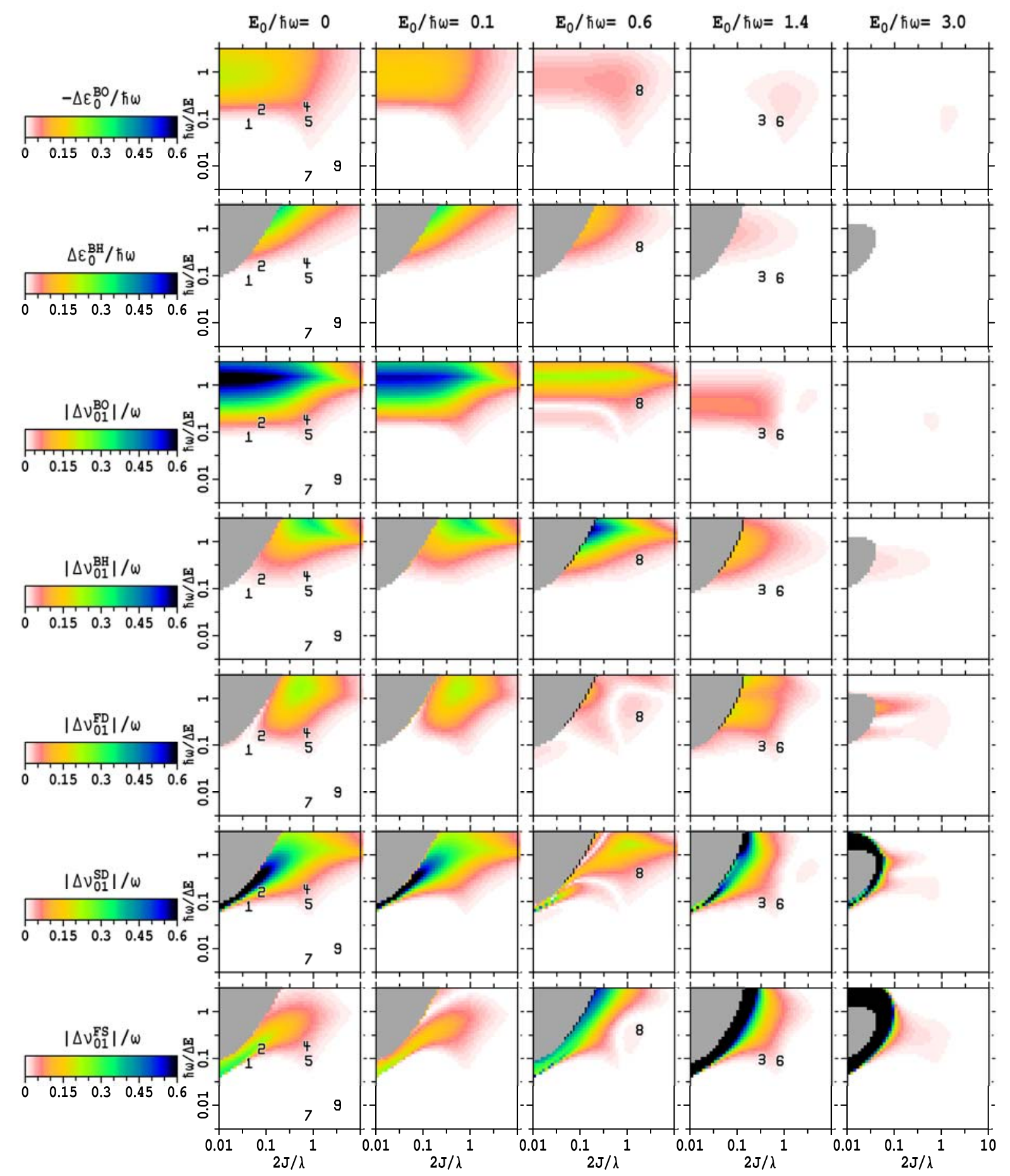

Fig. 6 The errors in the calculated lowest vibronic energy $\Delta \varepsilon_{0}$ (Eqn. (29)) and the absolute error in the lowest transition energy $\left|\Delta v_{01}\right|$ (Eqn. (30)) from the coupled diabatic harmonic-oscillator model are shown, evaluated using the BO (Eqn. (3)) approximation possibly corrected by just the DC (Eqn. (10), giving the Born-Huang adiabatic potential), or the FD or SD non-adiabatic coupling terms, or by both the FD and SD terms (FS); the grayed-out regions require $>1024$ vibrational basis functions for convergence. At low values of the asymmetry, all methods overestimate the transition energy. Sample model compounds 1-9 are indicated, see Table 1.

well $(2|J| / \lambda<1)$, decreasing steadily in magnitude as $E_{0} / \hbar \omega$ deviates from zero. They are small for most typical molecules ( $\hbar \omega / \Delta E<0.1$ ) but for such systems the maximum breakdown occurs in the strongly anharmonic transition region between 5 single-welled and double-welled systems $(2|J| / \lambda=1)$. However, Table 1 shows calculated values for the 10 sample molecules and the errors in the BO approximation can be significant, up to $5 \%$ of $\hbar \omega$ for the ground-state energy and $7 \%$ for the lowest vibrational transition energy.

10 A variety of approximate methods aimed at improving the BO approximation can be constructed by retaining one or two of the three neglected terms $\Delta H^{D C}, \Delta H^{F D}$, and $\Delta H^{S D}$ in Eqn. (7). Fig. 6 and Table 1 also show the error in the lowest energy level 
obtained when just $\Delta H^{D C}$ is included (the Born-Huang adiabatic approximation, Eqn. (10)), as well as the absolute errors in the lowest transition energy obtained using this method and by including only either $\Delta H^{F D}, \Delta H^{S D}$, or $\Delta H^{F D}+\Delta H^{S D}$.

As Eqn. (8) indicates that the DC correction to the BO potential energy surfaces is always positive (see e.g., Fig. 3), it always increases energy levels. Unlike the $\mathrm{BO}$ approximation, the Born-Huang approximation is variational and always overestimates the exact ground vibronic level's energy; this is 10 demonstrated in Table 1 where the calculated values of $\Delta \varepsilon_{0}^{B O}$ and $\Delta \varepsilon_{0}^{D C}$ are given for the sample molecules and characteristic data points. For all molecules considered, DC results in a significant improvement to the calculated transition energies, supporting the use of this approach in thermochemical

15 applications. Application of only the non-adiabatic FD and/or SD corrections always act to lower the calculated ground-state energy, thus always increasing the error compared to the BO approximation rather than reducing it. As Table 1 and Fig. 6 show, this scenario is typically maintained for the lowest

20 transition energy too, and this is the reason why DC is normally used in spectroscopy for the description of $\mathrm{BO}$ breakdown rather than the derivative corrections. However, the error in the BO approximation is quite small, $<5 \%$, in all of these cases, and for systems such as $\mathbf{A}$ the $\mathrm{BH}$ approximation is found to overestimate

25 the energy level by more than the $\mathrm{BO}$ approximation originally underestimated it. As the cusp diameter decreases and BO breakdown becomes large, using single Born-Huang adiabatic potential-energy surfaces becomes inadequate and the inclusion of the non-adiabatic correction terms is essential. In this regime

30 the effects of the three BO breakdown corrections are highly nonadditive, but even when the corrections are small, typically inclusion of all three terms is required for quantitative analysis. Some calculations in which these terms have been evaluated have noted this effect also. ${ }^{91}$

35 Useful analytical expressions may be derived for typical molecules with $\hbar \omega / \Delta E \ll 1$ and $E_{0}=0$. For single-well systems with $2|J| / \lambda>1$, the DC contribution decreases the force constants (see Eqn. (15)) decrease to

$$
\left.\frac{\partial^{2}}{\partial Q^{2}} \varepsilon_{ \pm}^{B H}(Q)\right|_{Q=0}=\hbar \omega\left(1 \pm \frac{\lambda}{2|J|}-\frac{1}{2}\left(\frac{\lambda}{2 J}\right)^{2}\left(\frac{\hbar \omega}{J}\right)^{2}\right)
$$

40 so that the associated Born-Huang vibration frequencies are given by

$$
\omega_{ \pm}^{B H}=\omega\left(1 \pm \frac{\lambda}{2|J|}-\frac{1}{2}\left(\frac{\lambda}{2 J}\right)^{2}\left(\frac{\hbar \omega}{J}\right)^{2}\right)^{1 / 2} .
$$

Double-well character persists in the Born-Huang potential up to

$$
\frac{2|J|}{\lambda}=\frac{1}{2}\left[1+\left(1+2\left(\frac{\hbar \omega}{\lambda}\right)^{2}\right)^{1 / 2}\right] .
$$

45 However, the location, energy correction, and force constant of the double-well minima cannot be expressed in closed form; while accurate expansions are given in ESI, the dominant contributions in the limits of $2|J| / \lambda \ll 1$ and $\hbar \omega / \Delta E \ll 1$ are:

$$
\begin{aligned}
& Q_{\min }^{B H} \approx \pm Q_{m}\left[1-\left(\frac{2 J}{\lambda}\right)^{2}\left(1-4\left(\frac{\hbar \omega}{\lambda}\right)^{2}\right)\right]^{1 / 2}, \\
& \varepsilon_{-}^{B H}\left(Q_{\min }^{B H}\right)-\varepsilon_{-}\left(Q_{\min }^{B O}\right) \approx \frac{\hbar^{2} \omega^{2} J^{2}}{\lambda^{3}}=\frac{\hbar \omega}{4}\left(\frac{2 J}{\lambda}\right)^{2}\left(\frac{\hbar \omega}{\lambda}\right), \text { and (34) } \\
& \omega^{B H} \approx \omega\left[1-\left(\frac{2 J}{\lambda}\right)^{2}\left(1-10\left(\frac{\hbar \omega}{\lambda}\right)^{2}\right)\right]^{1 / 2},
\end{aligned}
$$

50 respectively, revealing how DC acts to increase localization, increase the heat of formation, and increase molecular vibration frequencies compared to BO predictions ${ }^{162}$ (i.e., those obtained setting $\hbar \omega / \lambda \approx \hbar \omega / \Delta E=0$ in Eqn. (34)). In addition, DC raises the transition-state energy of the Born-Huang energy ${ }_{55}$ surface by $\Delta \Delta E^{\dagger}$ (Eqns. (22) and (23)) plus the much smaller correction $\varepsilon_{-}^{B H}\left(Q_{\min }^{B H}\right)-\varepsilon_{-}\left(Q_{\min }^{B O}\right)$.

d. Shortcomings of calculated energy-level spacings near the transition state evaluated using approximate BO-based methods.

60 In ESI an analysis is presented of the energy gap between the two vibronic levels closest in energy to that of any transition state. As the cusp diameter decreases, introducing the $\Delta H^{F D}$ and/or $\Delta H^{S D}$ corrections alone causes this energy gap to deviate much further from the "true" value than was produced by the original ${ }_{65} \mathrm{BO}$ approximation. Again we see that all three corrections must be applied in order to obtain robust and reliable results.

\section{e. Shortcomings of quantum dynamics evaluated using} approximate BO-based methods.

The rates for electron-transfer and other processes that are poorly 70 described by tunneling-corrected transition-state theory are most commonly modeled quantitatively using trajectories ran on multiple or mixed BO potential-energy surfaces, though diabatic surfaces have also been used. ${ }^{81,171}$ In general, a wide variety of ansatz are available for this, ${ }^{11,12,61,81,172-175}$ but here we evoke 75 none of these but instead focus on a simple enabling property- the quantum dynamics of a wavepacket running at the energy of the transition state. All methods for determining thermal rate expressions introduce the concept of temperature and then manipulate dynamics in some way, either by simple analytical 80 approximation or by using some modified classical, semiclassical, or quantum propagation scheme.

Quantum dynamics is solved by first diagonalizing the (possibly approximated) Hamiltonian operator, Eqn. (7), in the truncated vibrational basis. The time dependence of the 85 wavepacket is then expressed as

$$
|\Psi(r, Q ; t)\rangle=\sum_{i=1}^{2 N} d_{i}(t)\left|\psi_{i}(r, Q)\right\rangle
$$

where $d_{i}(0)$ is the projection of the initial coherent state

$$
|\Psi(r, Q ; 0)\rangle=\pi^{-1 / 4} \exp \left[-\left(Q+Q_{m}\right)^{2} / 2\right]\left|\phi_{-}^{B O}(r, Q)\right\rangle
$$

onto the eigenfunctions $\left|\psi_{i}(r, Q)\right\rangle$ of the Hamiltonian, Eqn. (12), 90 where

$$
d_{i}(t)=d_{i}(0) \exp \left(-i \varepsilon_{i} t / \hbar\right) .
$$

An alternative procedure would be to numerically solve the timedependent Schrödinger equation $i \hbar \mathrm{d}|\Psi\rangle / \mathrm{d} t=\mathbf{H}|\Psi\rangle, \quad$ a procedure that is usually computationally much more efficient as 

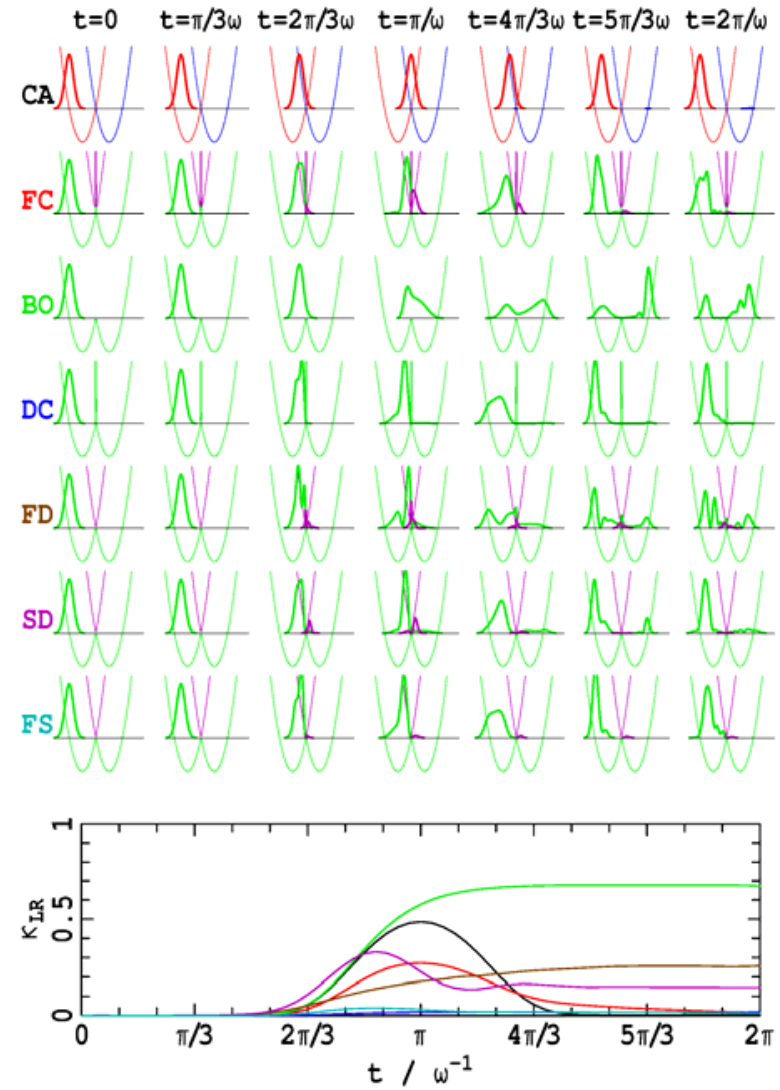

Fig. 7 Quantum dynamics of a coherent-state (if $J=0$ ) wavepacket starting at the far left of the left-hand BO well at the energy of the transition state for scenario $\mathbf{D}\left(2 J / \lambda=0.01, \hbar \omega / \Delta E=0.1, E_{0}=0\right)$.

The top frames show the wavepacket vibrational density resolved onto the electronic basis states (either CA, with propagation based on

Eqn. (1), or else BO, with propagation based on Eqn. (7)),

superimposed as appropriate on either the CA potential-energy surfaces (red- L, blue-R) or else one or two of the $\mathrm{BO}$ or Born-Huang surfaces (green- GS, magenta- ES) for different Hamiltonians. BO dynamics is based on either: full corrections $(\mathrm{FC}=\mathrm{DC}+\mathrm{FD}+\mathrm{SD}$, red), $\mathrm{BO}$ only (no corrections, green), using DC correction only (blue), FD correction only (brown), SD correction only (magenta), or

$\mathrm{FS}=\mathrm{FD}+\mathrm{SD}$ corrections (cyan). The lower figures show the fraction $\kappa_{L R}$ of the wavepacket located to the right of the transition as a function of time for one period of a vibration.

the propagation time used is very short, only one vibrational period. However, all numerical integrators that we used were unstable, displaying large Lyapunov exponents which induced rapid exponential divergence of numerical error as time 5 increased. Such phenomena are a known property of the Cusp Catastrophe. ${ }^{93-95}$

We choose the initial coherent-state wavepacket starting at the left-hand end of the BO surfaces sketched in Fig. 3 (essentially localized in the $\mathrm{L}$ diabatic well). This then runs towards the 10 transition-state region, with some of its contents passed through into the $\mathrm{R}$ well and some reflected back into the $\mathrm{L}$ well. We determine the probability $\kappa_{L R}$ of crossing the transition state on first attempt, evaluated as the density of the wavepacket found on the right-hand side of the transition state after one period ( $15 t=2 \pi / \omega)$ of motion; we do not attempt to analyze the results of multiple crossings as this would introduce severe wavepacket interference effects into the analysis, effects that would be highly dependent on features such as the number of included modes and dephasing. If the coupling between the diabatic states $J$ is zero, 20 then this probability is zero, the wavepacket moving coherently (i.e., without changing shape with its average position and momentum evolving according to Hamilton's classical equations of motion) ${ }^{176}$ from its starting position to slightly past the transition state and then back to its original position. Note that 25 our approach ignores possible decoherence phenomena associated with molecular vibrations not included in the single-mode model. ${ }^{177-179}$ Such decoherence would need to act faster than one vibrational period in the active mode for our analysis to be invalid, an unusual but not unprecedented scenario.

30 Fig. 7 shows the calculated quantum dynamics of this wavepacket for scenario $\mathbf{D}$ from Table 1 and Fig. 3 with $2 J / \lambda$ $=0.01, \hbar \omega / \Delta E=0.1$, and $E_{0}=0$. The top row of the figure shows the results of solving Eqn. (1) in the CA basis for a wavepacket initially localized on the BO ground-state (see later 35 in Eqn. (36), projected back onto the diabatic basis). Shown in the figure is the vibrational density of this wavepacket projected onto the L and R CA basis electronic states. At such a small value of $2 J / \lambda$, the wavepacket moves essentially coherently on the $\mathrm{L}$ surface and is reflected back from the transition state with a 40 penetration of only $\kappa_{L R}=0.0036$ at the end of the trajectory (intermediate values are plotted in the bottom panel of the figure). The second row shows the results of a similar calculation evaluated in the $\mathrm{BO}$ basis using the full set of all $3 \mathrm{BO}$ correction terms (FC) in Eqn. (7). Here the vibrational density is projected 45 onto the GS and ES BO electronic basis states. In principle the sum of the two projected densities in the first two rows should be equivalent, but only $N=1024$ harmonic-oscillator basis functions per electronic state are used with the $\mathrm{BO}$ basis for which adequate convergence is not yet obtained. The differences are small 50 enough to neglect for our purposes here (CA transmission is $0.036 \%, \mathrm{FC}$ is $1.4 \%$ ) but would be important for any molecular calculation performed in this region of the parameter space.

We now consider the impact of using approximate dynamics in the $\mathrm{BO}$ basis, including either one, two, or none of the BO 55 breakdown terms from Eqn. (7) in the calculations. Firstly row 3 of Fig. 7 shows the results of orthodox $\mathrm{BO}$ adiabatic dynamics, neglecting all three correction terms. The wavepacket approaches the transition state unhindered with just over half of it (that half with an energy in excess of the transition state) passing through 60 and one half being reflected. Results of this nature are imbedded into standard transition-state theory and fail to capture the critical impact that $\mathrm{BO}$ breakdown has on the dynamics.

The next row shows the results of similar adiabatic dynamics involving no surface hopping applying only the DC to enforce ${ }_{65}$ dynamics on the Born-Huang GS surface, Eqn. (10). As Fig. 3 indicates, DC adds a huge positive potential in the immediate vicinity of the transition state, raising the transition-state energy by $\Delta \Delta E^{\dagger}=\hbar \omega / 8 Q_{C}^{2}=\hbar^{2} \omega^{2} \lambda / 16 J^{2}=250 \hbar \omega$ (Eqns. (20-23)) to well beyond the average energy of the wavepacket (which is 70 centered at the BO transition-state energy of $\Delta E^{\dagger}=$ just $2.5 \hbar \omega$ (Eqn. (21)). This forces the wavepacket to reflect almost in its entirety into the $\mathrm{L}$ well, with tunneling through the high but narrow Born-Huang potential-energy barrier being responsible for the calculated small product yield of $1.8 \%$ (close to the 75 previous result from the full dynamics of $1.6 \%$ ). Thus the BornHuang dynamics closely mimics the exact dynamics yet does not involve surface hopping or other non-adiabatic effects.

The effects of the lowest-order non-adiabatic correction $\Delta H^{F D}$ acting alone are shown in row 5 of Fig. 7 . As the 80 wavepacket approaches the transition state, surface hopping 

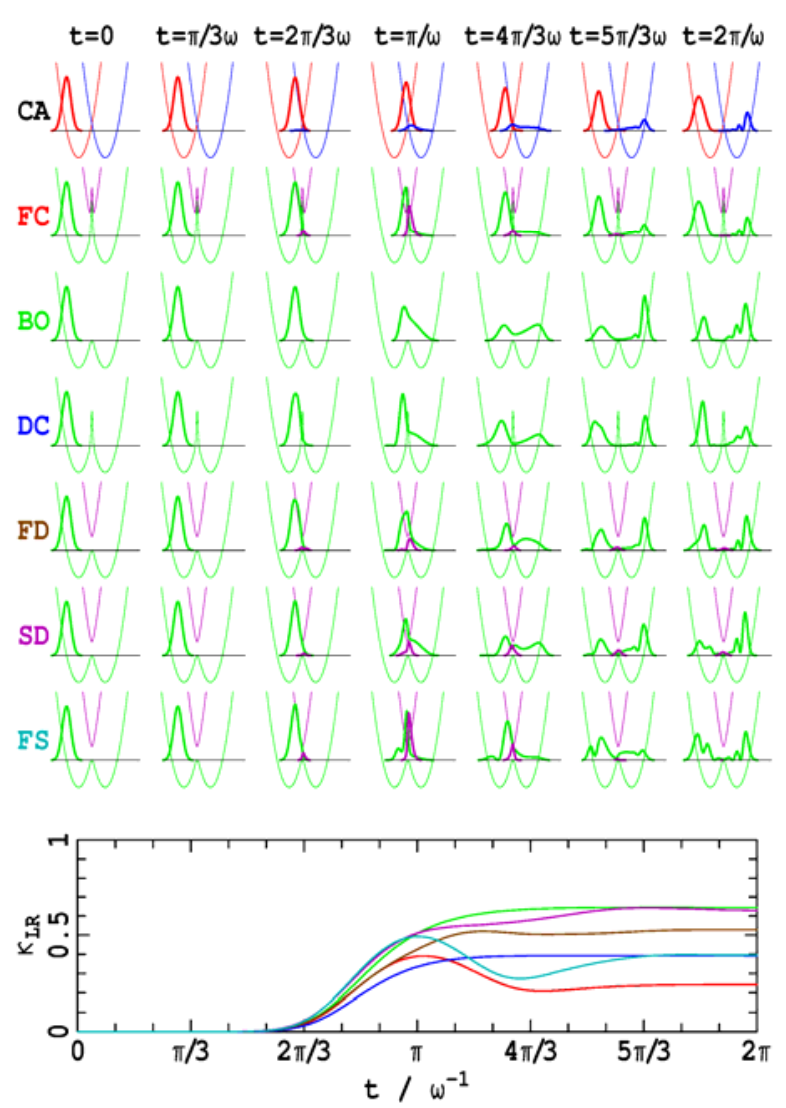

Fig. 8 Quantum dynamics of a coherent-state (if $J=0$ ) wavepacket starting at the far left of the left-hand $\mathrm{BO}$ well at the energy of the transition state for scenario $\mathbf{E}\left(2 J / \lambda=0.1, \hbar \omega / \Delta E=0.1, E_{0}=0\right)$. The top frames show the wavepacket vibrational density resolved onto the electronic basis states (either CA, with propagation based on

Eqn. (1), or else BO, with propagation based on Eqn. (7)),

superimposed as appropriate on either the CA potential-energy surfaces (red- L, blue-R) or else one or two of the $\mathrm{BO}$ or Born-Huang surfaces (green- GS, magenta- ES) for different Hamiltonians. BO dynamics is based on either: full corrections $(\mathrm{FC}=\mathrm{DC}+\mathrm{FD}+\mathrm{SD}$, red), $\mathrm{BO}$ only (no corrections, green), using DC correction only (blue), FD correction only (brown), SD correction only (magenta), or

$\mathrm{FS}=\mathrm{FD}+\mathrm{SD}$ corrections (cyan). The lower figures show the fraction $\kappa_{L R}$ of the wavepacket located to the right of the transition as a function of time for one period of a vibration.

occurs with high probability. Dynamics then proceeds on the ES until the classical turning point is reached, after which the wavepackets heads back down the ES potential towards the TS again, surface-hops back to the GS and into the L potential well, 5 leading to no net chemical reaction. This process is illustrated in Fig. 1 and is the standard mechanism by which deviations from transition-state theory are thought to arise. However, the calculations indicate that $26 \%$ of the wavepacket does not undergo this process but instead remains on the GS surface and

10 leads to product formation. Hence this approach fails to provide a useful quantitative description of the process. Uncharacteristically, the higher-order non-adiabatic correction $\Delta H^{S D}$ shown in row 6 seems to perform better, yielding just $15 \%$ products, with the two derivative couplings (FS) combining 15 to give a more realistic yield of $1.3 \%$. Interestingly, they do this by reducing the apparent instantaneous surface hoping. When all 3 correction terms are used the results are again highly nonadditive, with the $\mathrm{DC}$ and $\mathrm{FD}+\mathrm{SD}$ terms separately yielding transmissions of around $1.5 \%$, about the same yield as for all 3 20 terms combined.

The nature of the processes involved are revealed further by consideration of the results at $t=\pi / \omega$, a time just after the wavepacket impinges on the transition state. The exact dynamics (FC) and the dynamics on the Born-Huang potential25 energy surface (DC) show the same major qualitative feature with the maximum in the vibrational density never reaching the transition state. Naively, such blocking is not expected as DC arises $^{9,60}$ as the square of the FD correction (Eqn. (19)-(20)) and so is intrinsically narrower (e.g., at $Q=Q_{\mathrm{x}}+Q_{C}$, 30 $\Delta P^{F D} /\left(\Delta P^{F D}\right)_{\max }=1 / 2$ while $\left.\Delta H^{D C} /\left(\Delta H^{D C}\right)_{\max }=1 / 4\right)$, allowing surface hopping to occur before access is blocked. However, as $\Delta \Delta E^{\dagger} \gg \Delta E^{\dagger}$ the outer tail of the correction actually exceeds the energy of the wavepacket at distances well removed from the transition state, blocking motion. From Eqn. ${ }_{35}(8), \Delta \Delta E^{\dagger}=\hbar \omega$ at $\left|Q-Q_{\mathrm{X}}\right|=12 Q_{C}$, a geometry at which $\Delta P^{F D}(Q) /\left(\Delta P^{F D}\right)_{\max }=0.007$ (Eqns. (8) and (19)) and so, even allowing for tunneling through the thin DC spike, the impact of the momentum correction in reduced from what it might otherwise have been. Including the FD correction alone gives

$4013 \%$ of the wavepacket on the ES at $t=\pi / \omega$, while including only SD gives a higher value of $15 \%$; note that these are instantaneous values and that some of the density that had earlier crossed to the ES by this time has now re-crossed back to the GS. However, the complex nature of the interplay between all 3 45 correction terms is demonstrated by noting that FD+SD combined yields only $2 \%$ on the ES at this time, the two contributions acting to largely cancel each other out, while the full calculation $\mathrm{DC}+\mathrm{FD}+\mathrm{SD}$ yields $27 \%$ on the ES so that the FD and SD terms now appear to reinforce each other. It is clear that the FD and SD 50 terms enhance tunneling through the Born-Huang potential barrier and then funnel this density onto the ES by surface hoping, a feature that most likely is a legacy of the enhanced width of the FD function.

Fig. 8 shows corresponding plots for scenario $\mathbf{E}$ from Table 1 55 and Fig. 3 with $2 J / \lambda=0.1, \hbar \omega / \Delta E=0.1$, and $E_{0}=0$ for which there are no convergence problems when using the $\mathrm{BO}$ description. The much larger coupling opens up a substantial gap between the GS and ES at the transition state, increases considerably the cusp diameter and so increases the likelihood 60 that transition-state theory could adequately describe chemical reaction dynamics. This scenario is nevertheless close to the actual ones for the charge-transfer molecules 1 (DPP) and 2 (Alq3), systems that would normally be treated using nonadiabatic electron-transfer theory rather than transition-state 65 theory or some adiabatic variant. In the CA basis, $\kappa_{L R}=0.24$ of the wavepacket crosses from the $\mathrm{L}$ diabatic state to the $\mathrm{R}$ diabatic state at the transition state, yielding reaction products identified after one period of motion. Using the full Hamiltonian containing all 3 corrections in the $\mathrm{BO}$ basis (FC), a numerically 70 equivalent total density is obtained, but the partitioning of the wavepacket into the different electronic basis states is quite different with in this case the wavepacket returning to the BO GS at the end of the trajectory after a substantial fraction crossed to the ES and then back again to yield no net reaction. Using the BO 75 approximation run on the GS surface only, the fraction of products is much larger, 0.63, indicating that BO breakdown remains important. The FD-only approximation also seriously overestimates the product yield at $\kappa_{L R}=0.53$ while using the DC 
$t=0 \quad t=\pi / 3 \omega \quad t=2 \pi / 3 \omega \quad t=\pi / \omega \quad t=4 \pi / 3 \omega t=5 \pi / 3 \omega \quad t=2 \pi / \omega$

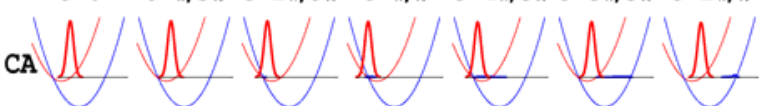
roW/W/WN/W/W/W oW WWWWWW $\because W W W W W W$

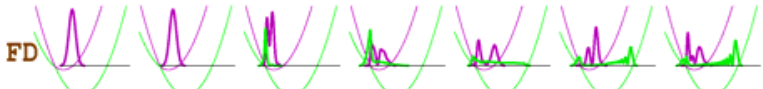
$\operatorname{so} W / W / W / W / W$ :DWWWNWW

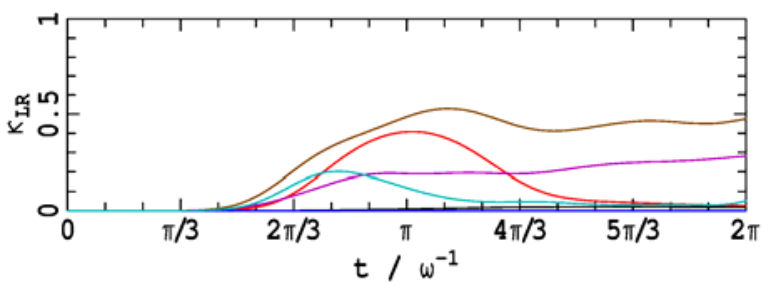

Fig. 9 Quantum dynamics of a coherent-state (if $J=0$ ) wavepacket starting at the far left of the left-hand $\mathrm{BO}$ well at the energy of the transition state for scenario $\mathbf{0}\left(\mathrm{FcPC}_{60}\right)(2 \mathrm{~J} / \lambda=0.029$,

$\left.\hbar \omega / \Delta E=0.15, E_{0} / \hbar \omega=-13\right)$. The top frames show the wavepacket vibrational density resolved onto the electronic basis states (either CA, with propagation based on Eqn. (1), or else BO, with propagation based on Eqn. (7)), superimposed as appropriate on either the CA potential-energy surfaces (red- L, blue-R) or else one or two of the BO or Born-Huang surfaces (green- GS, magenta- ES) for different Hamiltonians. BO dynamics is based on either: full corrections $(\mathrm{FC}=\mathrm{DC}+\mathrm{FD}+\mathrm{SD}$, red), $\mathrm{BO}$ only (no corrections, green), using DC correction only (blue), FD correction only (brown), SD correction only (magenta), or FS=FD+SD corrections (cyan). The lower figures show the fraction $\kappa_{L R}$ of the wavepacket located to the right of the transition as a function of time for one period of a vibration.

correction only gives the best yield of any approximate method, $39 \%$, with the low additional barrier height of $\Delta \Delta E^{\dagger}=2.5 \hbar \omega$ not being sufficient to reflect the whole wavepacket; using SD only the yield is $63 \%$, and while using $\mathrm{FD}+\mathrm{SD}$ yields $40 \%$, indicative again of serious interplay between these two derivative coupling contributions. The situation is thus similar to that shown in Fig. 7 for case D, with the Born-Huang adiabatic surfaces giving the best results of any approximate method with use of both derivative corrections being of similar quality but use 10 of only the FD correction yielding poor results. Quantitative results require the inclusion of all 3 correction terms

The fraction of the wavepacket transmitted after one period of vibration is shown in ESI over the whole parameter space used in this study. At no point in the parameter space at which

15 significant BO breakdown occurs does the application of any partial correction lead to quantitatively accurate results, though the DC-only and FD-only approaches can yield qualitatively useful information, with the error for DC-only typically being significantly less than that for FD-only. This is particularly true 20 for important charge-transfer systems such as 1 (DPP), 2 (Alq3), and $\mathbf{5}(\mathrm{CT})$, as well as for spectroscopically relevant systems such 4 (BNB) and 3 ( $\left.{ }^{3} \mathrm{PYR}\right)$.

Fig. 9 shows analogous dynamics for charge recombination in $0\left(\mathrm{FcPC}_{60}\right), 2 J / \lambda=0.029, \hbar \omega / \Delta E=0.15$, and $E_{0} / \hbar \omega=-13$.

${ }_{25}$ As Fig. 2 indicates, this process is highly exothermic and occurs in the "inverted" region, despite the absence of an adiabatic transition state. The initial wavepacket is launched on the upper surface towards the point of intersection of the two diabatic surfaces. The exact calculations show a small amount $\kappa_{L R}=2 \%$ 30 of the wavepacket undergoes surface hoping to the ground state during the first collision. From a broad perspective the BO and $\mathrm{BH}$ approximations, which predict no reaction at all, describe this scenario well; however, it is only the fraction that reacts that is of practical importance, a feature that both of these methods fail to 35 describe. Including only the FD term leads to a severe overestimate of the reactivity, however, predicting $48 \%$ reaction, while SD only predicts $28 \%$. Again, the central feature of the non-adiabatic reaction dynamics is that the FD and SD terms largely cancel each other out, with the FS approximation 40 predicting only $5 \%$ reactivity, and inclusion of all three terms is again required for quantitative accuracy. So, while the effects of $\mathrm{BO}$ breakdown on thermal reactions manifest quite differently for normal reactions over transition states from those of reactions in the "inverted" region (Fig. 1), the general conclusions reached

45 remain the same concerning the significance of all three coupling terms.

The other scenario depicted in Fig. 1 pertains to high-energy reactions above the conical-intersection for which single-mode models are inadequate. Nevertheless, in ESI the analogous so quantum dynamics of the 1-mode model is depicted. While the effects of the BO-breakdown terms again manifest themselves qualitatively differently, all three BO-breakdown terms are found to be essential for quantitative analysis. Indeed, the continued importance of the SD term in multi-dimensional simulations is 55 anticipated based on the known general relationships ${ }^{10,59-61}$ that specify the relative magnitudes of the FD and SD corrections, as well as the known properties of the Cusp Catastrophe.

f. Relationship between this quantum dynamics and the Landau-Zener and Golden Rule approaches for electron${ }_{60}$ transfer reactions.

We now introduce a primitive method, similar to approximations used in deriving analytical expressions for rate constants, for mapping the results from the previous quantum dynamics calculations onto the transmission coefficient $\kappa$ that is often used ${ }_{65}$ to describe the breakdown of transition-state theory. As only about one half of the incoming wavepacket has enough energy to cross the transition state in a classical fashion, assuming that all density that enters into the $\mathrm{R}$ well is immediately dephased, ${ }^{175}$ this transmission is approximated by

70

$$
\kappa=\min \left(1,2 \kappa_{L R}\right),
$$

and this function is shown in Fig. 10 evaluated at $E_{0}=0$; the comprehensive results presented in ESI demonstrate that the probability $\kappa_{L R}$ of a wavepacket crossing the transition state is highly independent of asymmetry and so the presented results are 75 taken to be generally indicative. Fig. 10 shows results evaluated using the CA basis that are compared to the numerically equivalent ones obtained using the full Hamiltonian in the $\mathrm{BO}$ basis. Whilst some numerical convergence issues with the BO calculations were apparent in Fig. 7, the broader picture displayed 

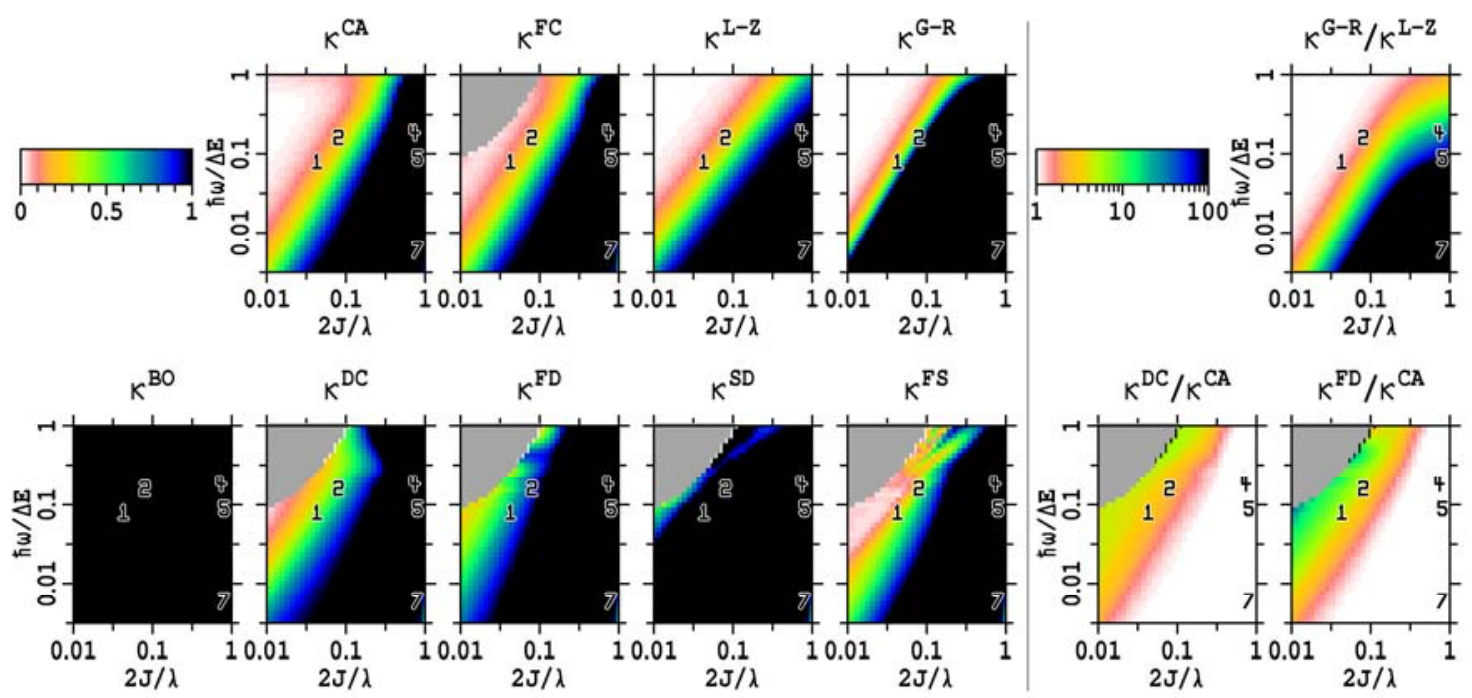

Fig. 10 The left-hand frames show the transmission coefficients for transition-state crossing estimated from one period of quantum wavepacket dynamics at the transition-state energy evaluated in the CA basis (Eqn. (1)) and the BO basis (Eqn. (7)) using the full Hamiltonian (FC) (i.e., all $\mathrm{DC}+\mathrm{FD}+\mathrm{SD}$ corrections), $\mathrm{BO}$ only, the DC correction only (Eqn. (10), the FD correction only, the SD correction only, or the FD+SD corrections are compared to those from Landau-Zener (L-Z) theory (Eqn. (42-44)) and its Golden-Rule (G-R) approximate (Eqn. (48)), both evaluated at $k_{\beta} T=\hbar \omega$. The right-hand frames show the ratios of some of these quantities on a log scale.

in Fig. 10 indicates that this effect is not qualitatively significant. Approximate transmission coefficients obtained using just none, one, or two of the BO-breakdown corrections are also shown in the figure. The BO approximation itself (no corrections used) has 5 unit transmission by definition whereas the transmissions predicted using only the DC or FD corrections are qualitatively similar to the exact results. To highlight the differences, the ratio of the transmissions predicted using only the FD or DC corrections to that from the exact calculations are also shown in

10 the figure, with results for the sample molecules also given in Table 1. The errors for the electron-transfer systems 1 (DPP) and 2 (Alq3) are significant, factors of 2-3 for DC-only and 3-4 for FD-only, demanding that all corrections be included in quantitative calculations but nevertheless indicating the utility of 15 the simpler methods for semi-quantitative analysis. Always the DC-only approximation gives more accurate results than does FD-only.

From a qualitative perspective, nonadiabatic chemical reactions are usually interpreted using Landau-Zener theory. ${ }^{102,}$ ${ }_{20} 103$ In this approach, the probability $P$ that a crossing of the transition state (by classical particles) starting on the ground-state BO surface leads to a product on the same surface is given by

$$
P=1-\exp \left(\frac{-2 \pi J^{2}}{\hbar v\left|\partial \varepsilon^{+} / \partial Q-\partial \varepsilon^{-} / \partial Q\right|}\right)
$$

where $\mathrm{v}$ is the speed at which the transition-state (treated as a 25 conical intersection) is crossed; ${ }^{11}$ there is, however, some ambiguity concerning the scaling of the exponent in this equation, with other modern sources quoting $\pi / 2$ rather than $2 \pi .^{12}$ For two diabatic states coupled through a single mode, Eqn. (39) evaluates to ${ }^{180}$

$$
{ }_{30} \quad P=1-\exp \frac{-\pi J^{2}}{\delta \mathrm{V}} .
$$

We evaluate this probability by averaging ${ }^{181}$ the velocities over a thermal distribution at temperature $T$

$$
\langle P\rangle=\int_{0}^{\infty} P \exp \frac{-\mathrm{v}^{2}}{2 k_{\beta} T} d \mathrm{v} / \int_{0}^{\infty} \exp \frac{-\mathrm{v}^{2}}{2 k_{\beta} T} d \mathrm{v} .
$$

but this integral is usually approximated instead using ${ }^{182}$$$
35 \quad\langle P\rangle \approx 1-\exp \frac{-\pi^{3 / 2} J^{2}}{\hbar \omega\left(\lambda k_{\beta} T\right)^{1 / 2}}
$$

although again the scaling factor is more frequently ${ }^{180}$ given as $\pi$ rather than $\pi^{3 / 2} \sim 5.6$; numerically, we find the optimum value to be 7.5 , a value that fits the numerically integrated results over the parameter space considered to an accuracy of $3.4 \%$ over a wide 40 temperature range. In terms of transition-state theory, nonadiabatic reaction-rate constants $k$ can be modeled using

$$
k=\kappa \frac{k_{\beta} T}{h} \exp \frac{-E^{\dagger}}{k_{\beta} T}=\kappa \frac{\omega}{2 \pi} \exp \frac{-E^{\dagger}}{k_{\beta} T}
$$

if one regards a possible reactive collision as happening once per molecular vibration, ${ }^{181}$ where the non-adiabaticity parameter $\kappa$ is ${ }_{45}$ given by ${ }^{92,180}$

$$
\kappa=\frac{2\langle P\rangle}{1+\langle P\rangle} .
$$

This property is also shown in Fig. 10 where it is compared to the analogous quantity determined from the trajectory propagations. Qualitatively, the results of the full quantum dynamics using all ${ }_{50}$ three BO correction terms are in good agreement with the standard Landau-Zener result, whereas using any approximate Hamiltonian gives poor results. Empirically the results in Fig. 10 can be represented using the simple function

$$
\kappa \approx \frac{c\left|\frac{2 J}{\lambda}\right|^{\alpha}\left(\frac{\hbar \omega}{\Delta E}\right)^{\beta}}{1+c\left|\frac{2 J}{\lambda}\right|^{\alpha}\left(\frac{\hbar \omega}{\Delta E}\right)^{\beta}}
$$

55 where we fit $c=3.22, \alpha=7, \quad \beta=-4$ to the results from the trajectory propagation and $c=1.63, \alpha=4, \beta=-3$ to the LandauZener results. In ESI, the fitted data is compared to the original, 
with the RMS errors found to be 5\% (trajectory data) and 2\% (Landau-Zener). Some significant quantitative differences between the trajectory results and the Landau-Zener expression are thus found. An alternative simpler empirical expression that 5 describes the trajectory calculations to the same accuracy (see ESI) is

$$
\kappa \approx \frac{\left(Q_{C} / 0.056\right)^{3}}{1+\left(Q_{C} / 0.056\right)^{3}},
$$

highlighting the critical role played by the cusp diameter in determining reaction kinetics. According to this equation, the 10 rate constant halves whenever $Q_{c}=0.056$, a value much smaller than the value of $Q_{c}=0.35$ previously identified as the largest value of the cusp radius for which the influence of DC on the activation energy was negligible, stressing the importance of this term to the reactivity.

15 In the weak-coupling limit of $\pi^{3 / 2} J^{2} / \hbar \omega\left(\lambda k_{\beta} T\right)^{1 / 2} \ll 1$, Eqn. (42) reduces to the standard Golden-Rule expression for the rate constant ${ }^{107,154,155}$

$$
k=\frac{2 \pi J^{2}}{\hbar \sqrt{4 \pi \lambda k_{\beta} T}} \exp \frac{-E^{\dagger}}{k_{\beta} T},
$$

so that the transmission coefficient becomes

$$
\begin{aligned}
\kappa & =\frac{2 \pi^{3 / 2} J^{2}}{\hbar \omega \sqrt{\lambda k_{\beta} T}}=\pi^{3 / 2}\left(\frac{\lambda}{k_{\beta} T}\right)^{1 / 2} Q_{c}^{2} \\
& \approx \frac{\pi^{3 / 2}}{8}\left(\frac{\hbar \omega}{k_{\beta} T}\right)^{1 / 2}\left(\frac{\Delta E}{\hbar \omega}\right)^{1 / 2} \frac{\hbar \omega}{\Delta \Delta E^{\dagger}}
\end{aligned}
$$

Hence the transmission is reduced as the temperature increases and as the ratio of the vibrational to electronic energy spacings increase but most importantly scales with the square of the cusp diameter and therefore inversely with the magnitude of the Born${ }_{25}$ Huang correction to the transition-state energy, Eqn. (19). This is why it is not possible to have a significant correction to the transition-state theory rate constant without the DC being important. Naively, the effect of the increased activation energy $\Delta \Delta E^{\dagger}=(\hbar \omega)^{2} \lambda / 16 J^{2}$ would be expected to decrease the rate 30 constant exponentially but tunneling through the rather narrow associated barrier, combined with the effects of surface hopping, reduce this dependence to only inverse proportionality.

Fig. 10 also shows the Golden-Rule rate from Eqn. (47) as well as the ratio of this value to that obtained using the Landau-Zener 35 method, exposing the consequences of use of the small-coupling approximation; ratios for the sample molecules and test systems with transition states are given in Table 1 . This ratio can exceed 1000 when the transmission is high but for $\mathbf{1}$ (DPP) and $\mathbf{2}$ (Alq3) it is only 1.2. Nevertheless, in Alq3 crystal there exists electron40 transfer couplings of much larger magnitudes than that for the fastest hole-transfer process considered herein and indeed for these pathways the Golden Rule expression does introduce multi order-of-magnitude errors. Similar effects have also been observed for other molecular conductors. ${ }^{173}, 174$ On a broader ${ }_{45}$ perspective, we have previously identified ${ }^{165,183} 10$ significant issues with the calculation of charge mobilities in organic conductors like Alq3, noting that realistic values typically result from the cancellation of many order-of-magnitude errors stemming from different aspects of the computational procedure.

\section{so 5. Conclusions}

This work is based on a simple model Hamiltonian that qualitatively accounts for a wide range of chemical thermodynamic, spectroscopic and kinetic processes in molecules, biological systems, and functional materials. Using it, 55 we demonstrate that, when the BO approximation performs poorly, it is usually necessary to treat all three contributions to BO breakdown so as to achieve quantitative results. This is demonstrated for:

- the lowest energy-level of the molecule and hence

60 thermochemical properties,

- vibration eigenstates at the bottom of deep potential-energy wells,

- the lowest-energy vibrational transitions,

- tunneling transitions,

65 - vibrational eigenstates in the vicinity of the transition state and for the spectroscopic transitions between them,

- quantum dynamics mimicking thermal reactions over a barrier, and

- quantum dynamics mimicking thermal reactions in the 70 "inverted" region where there is no transition state.

Use of the diagonal-correction (DC)-only correction (to produce the Born-Huang potential energy surface) is shown to be appropriate in some regions of the parameter space for groundstate thermochemistry, vibrational spectroscopy, and thermal 75 reactions including electron-transfer processes but use of first derivative (FD)-only methods is found never to be adequate. The DC term will contribute significantly to ground-state kinetic processes whenever the Born-Huang transition-state energy change $\Delta \Delta E^{\dagger}$ (Eqn. (21-23)) is large compared to the available 80 reaction energy. It blocks access to the transition state to inhibit surface hopping, leading to no reaction in the case of say thermal isomerization or electron-transfer processes. For thermochemical or spectroscopic data pertinent to the bottom of deep wells, the $\mathrm{DC}$ correction is well known to be the dominant one owing to the 85 large energy gap between the BO ground-state and excited-state which inhibits non-adiabatic interactions. Our extensions of this result to include many other processes including thermal kinetics provide a generalization of the conclusions drawn recently by Kutznelnigg ${ }^{62}$ for $\mathrm{H}_{2}{ }^{+}$, establishing this as a general aspect of 90 ground-state phenomena. Such a generalization is consistent with implications of the known fundamental relationships ${ }^{10,59-61}$ that related the three $\mathrm{BO}$-correction terms for problems involving multiple vibrational motions, conical intersections, and multiple electronic states. In general, however, quantitatively accurate 95 results require the inclusion of all three correction terms as the magnitudes of the smaller terms remain significant. Further, the effects of the three individual terms are non-additive, with often one minor-looking contribution acting to switch off more majorlooking ones.

100 Three key qualitative conclusions flow from this concerning the language that is in normal use concerning $\mathrm{BO}$ breakdown and chemical processes:

- thermal reactions that occur much slower than (tunneling corrected) transition-state theory predicts are usually termed

105 "non-adiabatic" reactions that are perceived as being blocked owing to non-adiabatic transitions to/from excited states, but as the DC term better accounts for the low rates and does not involve surface hoping, these reactions should only be termed "non-Born-Oppenheimer" ones. This in particular applies to weakly-coupled charge-transfer processes. Indeed, recent research $^{184-186}$ is highlighting the principle ${ }^{187}$ that it is always possible to describe such chemical process exactly using an adiabatic description. However, it is conceptually most helpful to still consider thermal reactions in the "inverted regime" and many high-energy photochemical processes to be "nonadiabatic" as the reactants and products are well represented by different adiabatic potential-energy surfaces. 
- provided that full quantum descriptions of molecular motion on a single potential-energy surface are always used, Born-Huang adiabatic potential-energy surfaces are in general more useful than Born-Oppenheimer ones, providing a significantly improved picture of chemical thermodynamics, vibration spectroscopy, and kinetics whilst retaining key chemical features such as the concepts of molecular vibration frequencies and transition-state energies.

- the properties of BO breakdown should be linked to the general

10 description of the Cusp Catastrophes as all identified major qualitative features stem from this primary origin. Dynamics around cusps is typically unstable owing to large Lyapunov exponents, and the results of trajectory propagations are known to be extremely sensitive to the initial conditions and approximations used. Further, the cusp diameter is identified as the critical physical property controlling BO breakdown based on simple analytical expressions (Eqn. (19-20)) that relate the three BO breakdown terms to each other and establish their close connection and common origin in terms of

20 the cusp diameter. Indeed, deviations of rate constants from the predictions of transition-state theory are also shown to correlate well with the cusp diameter (Eqn. (45)). These links developed between analytically solvable properties of the single-mode two-state model are of fundamental importance

25 because the anticipated consequences stemming from the mathematics of the cusp catastrophe are completely general in nature and underpin all effects of BO breakdown including the properties of conical intersections.

Unfortunately, with a few exceptions, ${ }^{66-74}$ most quantum 30 chemistry codes evaluate directly only the first-derivative coupling, making difficult practical calculations utilizing all molecular motions and all three contributions to BO breakdown. Our analytical expressions, however, allow for a diabatic approximation to be introduced in a non-critical way and utilized

35 only to determine the DC correction and the diagonal component of the SD corrections from the directly evaluated FD term using Eqn. (19). This may provide a practical computational method allowing for the inclusion of all three terms if the dynamics is tightly confined to be within a single vibrational motion. Most

40 significantly, these equations provide a specific application of generally known principles relating the three BO-breakdown corrections to each other. ${ }^{10,59-61}$ From a priori calculations of the FD term, the cusp diameter should be readily determinable, and from this Eqn. (45) even offers a direct route to quantum-kinetics 45 outcomes. However, photochemical applications will require extension of these equations to include different force constants for each diabatic state. Other ways of quickly estimating the DC term have also been proposed. ${ }^{71-73}$

Not surprisingly, the BO approximation itself is shown to be a 50 very good approximation over much of the parameter space accessible by normal molecular systems, i.e., those with low ratios of $\hbar \omega / \Delta E<0.1$ with the vibration energy spacing significantly smaller than the electronic spacing. In this regime highly improbable processes can be induced by the BO55 breakdown contributions, however, and so despite the approximation working very well, it may not describe some particular process of interest. In general it breaks down very quickly as $\hbar \omega / \Delta E$ increases above 0.1 or as the electronic coupling becomes much less than the reorganization energy, ${ }_{60} 2|J| / \lambda<0.1$. Quite surprisingly, we see that the BO description remains somewhat useful for the descriptions of the kinetics of many charge-transfer processes, particularly those of technological relevance such as Alq3 in molecular conductors as such applications are driven by the desire to have large electronic
${ }_{65}$ couplings and hence fast reaction rates. Recently, ${ }^{165}$ we reviewed standard calculation practices in this field, identifying a variety of critical issues with the calculation methods used each of which introduces order-of-magnitude errors yet the final computational strategy typically delivers good agreement with experiment. The 70 use of the Golden-Rule perturbation expression of non-adiabatic electron-transfer theory rather than more apt approaches such as transition-state theory was indeed identified as one of the critical issues. Additional circumstances with $2|J| / \lambda<0.1$ in which the

BO approximation and Golden-Rule expressions are likely to fail 75 include those involving synchronously coupled processes such as coupled proton-electron transfer reactions, ${ }^{188}$ processes for which the single-mode two state model is inappropriate.

The use of perturbation expressions for simple one-step electron-transfer processes has a long history stemming from the 80 development of applications in chemistry by Marcus during the 1950 's-60's of methods originally designed to describe highly improbable processes in physics that occur because of very weak coupling between [diabatic] states. ${ }^{189-191}$ They could be coupled with the use of transition-state theory for the pure diabatic ${ }^{85}$ scenario to yield approaches valid in both chemical extremes. ${ }^{192}$ Instead, following London, ${ }^{6}, 104$ Hush developed a fully general electron transfer theory, examining the adiabatic surfaces produced from coupled diabatic states, focusing on the chemical properties of the transition-state cusp as well as properties could 90 be directly determined using optical spectroscopy, quantum chemistry, and related methods. ${ }^{166-169}$ These quantum molecular properties do not enter into the alternative perturbation expressions, though Marcus did propose "fictitious charges" as a possible explanatory device for the properties of electron-transfer 95 transition states. ${ }^{190-192}$ We see here that adiabatic approaches are in general required for quantitative understanding of the properties of all types of charge-transfer systems, but especially those displaying the types of fast reaction rates desired for modern device applications.

\section{${ }_{100}$ Acknowledgments}

We thank the Australian Research Council Discovery Projects scheme for funding, National Computational Infrastructure (NCI) for providing computational resources, and Profs. John Stanton, Meredith Jordan and Timothy Schmidt for helpful discussions.

105

\section{Notes and references}

a: International Centre for Quantum and Molecular Structure, College of Sciences, Shanghai University, Shanghai 200444 China; Tel: 86-

15618155341; E-mail: Jeffrey.Reimers@uts.edu.au

110 b: School of Mathematical and Physical Sciences, University of Technology Sydney, NSW 2007 Australia

c: c: Department of Physics and Astronomy, University College London, Gower Street, London WC1E 6BT UK

d: School of Chemistry, The University of Sydney, Sydney, NSW 2006

115 Australia

e: School of Mathematics and Physics, The University of Queensland, QLD 4072 Australia

f: School of Molecular Biosciences, The University of Sydney, NSW, 2006 Australia

120

$\dagger$ Electronic Supporting Information (ESI) available: S1. The use of a single-mode two-state model to describe complex molecular systems; S2. More accurate expressions for properties of the Born-Huang adiabatic surface; S3. Shortcomings of calculated energy-level spacings near the 125 transition state evaluated using approximate BO-based methods; S4. The fraction of the wavepacket depicting thermal reactions transmitted after one period of motion; S5. Approximating the transmission coefficient; S6. High-energy dynamics above the conical intersection as is pertinent

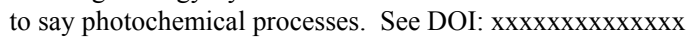


1. M. Born and R. Oppenheimer, Ann. Phys., 1927, 84, 0457.

2. M. Born, Nachr. Akad. Wiss. Gottingen, Math.-physik. Klasse IIa, Math.-physik.-chem. Abt., 1951, 1.

5 3. T. Azumi and K. Matsuzaki, Photochem. Photobiol., 1977, 25, 315.

4. B. T. Sutcliffe and R. G. Woolley, J. Chem. Phys., 2012, 137, $22 \mathrm{~A} 544$.

5. W. Heitler and F. London, Z. Phys., 1927, 44, 455.

6. F. London, in Probleme der Modernen Physik, ed. P. Debye, Hirsel, Leipzig, 1928, p. 104

7. H. Eyring and M. Polanyi, Z. Phys. Chem. B, 1931, 12, 279.

8. J. C. Tully, Modern Methods for Multidimensional Dynamics Computations in Chemistry, 1998.

9. A. W. Jasper, B. K. Kendrick, C. A. Mead and D. G. Truhlar, Adv. Ser. Phys. Chem., 2004, 14, 329.

10. A. W. Jasper and D. G. Truhlar, Adv. Ser. Phys. Chem., 2011, 17, 375.

11. J. C. Tully, J. Chem. Phys., 2012, 137, 22 A301.

12. T. Yonehara, K. Hanasaki and K. Takatsuka, Chem. Rev., 2012, 112, 499.

13. A. de la Lande, J. Řezáč, B. Lévy, B. C. Sanders and D. R. Salahub, J. Am. Chem. Soc., 2011, 133, 3883.

14. A. de la Lande, N. S. Babcock, J. Rezac, B. Levy, B. C. Sanders and D. R. Salahub, Phys. Chem. Chem. Phys., 2012, 14, 5902.

25 15. U. Manthe, H. D. Meyer and L. S. Cederbaum, J. Chem. Phys., 1992, 97, 3199 .

16. Y. Shigeta, H. Nagao, K. Nishikawa and K. Yamaguchi, J. Chem. Phys., 1999, 111, 6171.

17. R. Longo, A. Diz, E. Deumens and Y. Öhrn, Chem. Phys. Lett., 1994, 220, 305 .

18. E. Deumens and Y. Öhrn, J. Phys. Chem. A, 2001, 105, 2660.

19. G. A. Worth and L. S. Cederbaum, Annu. Rev. Phys. Chem., 2004, $\mathbf{5 5}, 127$.

20. N. L. Guevara, E. Teixeira, B. Hall, Y. Öhrn, E. Deumens and J. R. Sabin, Phys. Rev. A, 2011, 83, 052709.

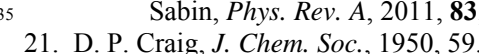

22. P. Saxe, B. H. Lengsfield, III and D. R. Yarkony, Chem. Phys. Lett., $1985,113,159$.

23. T. Vertesi, A. Vibok, G. J. Halasz, A. Yahalom, R. Englman and M. Baer, J. Phys. Chem. A, 2003, 107, 7189.

24. S. Nangia and D. G. Truhlar, J. Chem. Phys., 2006, 124, 124309.

25. J. Li, C.-K. Lin, X. Y. Li, C. Y. Zhu and S. H. Lin, Phys. Chem. Chem. Phys., 2010, 12, 14967.

26. J. Loreau, J. Lievin, P. Palmeri, P. Quinet and N. Vaeck, J. Phys. B:

At., Mol. Opt. Phys., 2010, 43, 065101.
27. G. Capecchi and H.-J. Werner, Phys. Chem. Chem. Phys., 2004, 6, 4975.

28. R. Valero, L. Song, J. Gao and D. G. Truhlar, J. Chem. Theory Comput., 2009, 5, 1.

50 29. K. Saita and D. V. Shalashilin, J. Chem. Phys., 2012, 137, 22 A506.

30. M. Bockmann, N. L. Doltsinis and D. Marx, J. Chem. Phys., 2012, 137, 22A505

31. R. R. Valiev, V. N. Cherepanov, V. Y. Artyukhov and D. Sundholm, Phys. Chem. Chem. Phys., 2012, 14, 11508.

55 32. F. Plasser, G. Granucci, J. Pittner, M. Barbatti, M. Persico and H. Lischka, J. Chem. Phys., 2012, 137, 22A514.

33. B. R. L. Galvao, P. J. S. B. Caridade and A. J. C. Varandas, J. Chem. Phys., 2012, 137, 22A515.

34. X. Zhu and D. R. Yarkony, J. Chem. Phys., 2012, 137, 22 A511.

60 35. W. D. Hobbey and A. D. McLachlan, J. Chem. Phys., 1960, 33, 1695 .

36. W. Lichten, Phys. Rev., 1963, 131, 229.

37. T. F. O'Malley, Phys. Rev., 1967, 162, 98.

38. F. T. Smith, Phys. Rev., 1969, 179, 111.

65 39. V. Sidis and H. Lefebvre-Brion, Journal of Physics B: Atomic and Molecular Physics, 1971, 4, 1040.

40. M. Gouterman, J. Chem. Phys., 1965, 42, 351.

41. L. Ziegler and A. C. Albrecht, J. Chem. Phys., 1974, 60, 3558.

42. I. G. Ross, Isr. J. Chem., 1975, 14, 118.

70 43. G. Fischer, J. R. Reimers and I. G. Ross, Chem. Phys., 1981, 62, 187.

44. P. J. Chappell, G. Fischer, J. R. Reimers and I. G. Ross, J. Molec. Spectrosc., 1981, 87, 316.

45. D. R. Yarkony, Rev. Mod. Phys., 1996, 68, 985.
46. D. R. Yarkony, Acc. Chem. Res., 1998, 31, 511.

75 47. Z.-L. Cai and J. R. Reimers, J. Phys. Chem. A, 2000, 104, 8389.

48. H. Nakamura and D. G. Truhlar, J. Chem. Phys., 2002, 117, 5576.

49. J. R. Reimers and N. S. Hush, J. Chem. Phys., 2003, 119, 3262.

50. J. R. Reimers and N. S. Hush, Chem. Phys., 2004, 299, 79.

51. T. Ichino, S. W. Wren, K. M. Vogelhuber, A. J. Gianola, W. C. 80 Lineberger and J. F. Stanton, J. Chem. Phys., 2008, 129, $084310 / 1$.

52. T. Ichino, J. Gauss and J. F. Stanton, J. Chem. Phys., 2009, 130.

53. K. Pachucki and J. Komasa, J. Chem. Phys., 2009, 130, 164113.

54. T. Van Voorhis, T. Kowalczyk, B. Kaduk, L.-P. Wang, C.-L. Cheng and Q. Wu, Annu. Rev. Phys. Chem., 2010, 61, 149.

55. N. Zamstein and D. J. Tannor, J. Chem. Phys., 2012, 137, 22 A517.

56. T. Pacher, L. S. Cederbaum and H. Koeppel, J. Chem. Phys., 1988, 89, 7367.

57. M. Born and K. Huang, Dynamical Theory of Crystal Lattices,

Clarendon, Oxford, 1954.
58. S. L. Mielke, D. W. Schwenke, G. C. Schatz, B. C. Garrett and K. A. Peterson, J. Phys. Chem. A, 2009, 113, 4479.

59. T. C. Thompson, D. G. Truhlar and C. A. Mead, J. Chem. Phys., $1985,82,2392$.

95 60. B. K. Kendrick, C. A. Mead and D. G. Truhlar, Chem. Phys., 2002, 277, 31 .

61. C. Zhu, A. W. Jasper and D. G. Truhlar, J. Chem. Phys., 2004, 120, 5543.

62. W. Kutzelnigg, Mol. Phys., 2007, 105, 2627.

100 63. W. Kolos and L. Wolniewicz, J. Chem. Phys., 1968, 49, 404.

64. B. C. Garrett and D. G. Truhlar, J. Chem. Phys., 1985, 82, 4543.

65. S. L. Mielke, D. W. Schwenke and K. A. Peterson, J. Chem. Phys., 2005, 122, 224313

66. N. C. Handy, Y. Yamaguchi and H. F. Schaefer Iii, J. Chem. Phys., 1986, 84, 4481.

67. J. O. Jensen and D. R. Yarkony, J. Chem. Phys., 1988, 89, 975.

68. E. F. Valeev and C. D. Sherrill, J. Chem. Phys., 2003, 118, 3921

69. J. Gauss, A. Tajti, M. Kallay, J. F. Stanton and P. G. Szalay, J. Chem. Phys., 2006, 125, 144111.

110 70. H.-J. Werner, M. Kallay and J. Gauss, J. Chem. Phys., 2008, 128, 034305/1.

71. J. R. Mohallem, T. d. O. Coura, L. G. Diniz, C. G. de, D. Assafrao and T. Heine, J. Phys. Chem. A, 2008, 112, 8896.

72. J. R. Mohallem, J. Chem. Phys., 2008, 128, 144113/1.

115 73. A. Tajti, P. G. Szalay and J. Gauss, J. Chem. Phys., 2007, 127, 014102/1.

74. T. D. Crawford, C. D. Sherrill, E. F. Valeev, J. T. Fermann, R. A. King, M. L. Leininger, S. T. Brown, C. L. Janssen, E. T. Seidl, J. P. Kenny and W. D. Allen, J. Comput. Chem., 2007, 28, 1610 .

75. M. E. Harding and W. Klopper, ChemPhysChem, 2013, 14, 708

76. M. Przybytek and B. Jeziorski, Chem. Phys., 2012, 401, 170.

77. M. Pavanello, L. Adamowicz, A. Alijah, N. F. Zobov, I. I. Mizus, O. L. Polyansky, J. Tennyson, T. Szidarovszky and A. G. Csaszar, J. Chem. Phys., 2012, 136, 184303/1.

78. N. J. DeYonker and W. D. Allen, J. Chem. Phys., 2012, 137, 234303/1.

79. U. Bozkaya, J. M. Turney, Y. Yamaguchi and H. F. Schaefer, III, J. Chem. Phys., 2012, 136, 164303/1.

130 80. A. Yachmenev, S. N. Yurchenko, T. Ribeyre and W. Thiel, J. Chem. Phys., 2011, 135, 074302/1.

81. T. R. Rao and S. Mahapatra, J. Chem. Phys., 2011, 134, 204307/1.

82. P. Meier, M. Neff and G. Rauhut, J. Chem. Theory Comput., 2011, 7, 148.

135 83. J. Lievin, J. Demaison, M. Herman, A. Fayt and C. Puzzarini, J. Chem. Phys., 2011, 134, 064119/1.

84. F. Holka, P. G. Szalay, J. Fremont, M. Rey, K. A. Peterson and V. G. Tyuterev, J. Chem. Phys., 2011, 134, 094306/1.

85. W. Klopper, R. A. Bachorz, D. P. Tew and C. Hattig, Phys. Rev. A: At., Mol., Opt. Phys., 2010, 81, 022503/1.

At., Mol., Opt. Phys., 2010, 81, 022503/1.
86. A. Karton and J. M. L. Martin, J. Chem. Phys.. 2010, 133, 144102.

87. U. Bozkaya, J. M. Turney, Y. Yamaguchi and H. F. Schaefer, III, J. Chem. Phys., 2010, 132, 064308/1.

88. S. L. Mielke, D. W. Schwenke, G. C. Schatz, B. C. Garrett and K. A. 145 Peterson, J. Phys. Chem. A, 2009, 113, 4479. 
89. S. L. Hobson, E. F. Valeev, A. G. Csaszar and J. F. Stanton, Mol. Phys., 2009, 107, 1153.

90. S. Hirata, E. B. Miller, Y.-y. Ohnishi and K. Yagi, J. Phys. Chem. A, 2009, 113, 12461

5 91. D. W. Schwenke, J. Phys. Chem. A, 2001, 105, 2352.

92. M. D. Newton and N. Sutin, Annu. Rev. Phys. Chem., 1984, 35, 437.

93. P. T. Saunders, An introduction to catastrophe theory, Cambridge University Press, Cambridge, 1980.

94. F. Xu, Z. Phys. Chem., 1990, 166, 79

10 95. X. Krokidis, B. Silvi, C. Dezarnaud-Dandine and A. Sevin, New J. Chem., 1998, 22, 1341.

96. D. N. Beratan and J. J. Hopfield, J. Chem. Phys., 1984, 81, 5753.

97. J. N. Onuchic and P. G. Wolynes, J. Phys. Chem., 1988, 92, 6495

98. S. Larsson, Int. J. Quantum Chem., 1982, 22, 385.

15 99. N. S. Hush, J. Polymer Sci., 1953, 11, 289.

100.P. Politzer, J. R. Reimers, J. S. Murray and A. Toro-Labbe, J. Phys. Chem. Lett., 2010, 1, 2858.

101.L. D. Landau, Z. Phys. Sowjetunion, 1932, 1, 88.

102.L. D. Landau, Z. Phys. Sowjetunion, 1932, 2, 46.

20 103.C. Zener, Proc. R. Soc. London, Ser. A, 1932, 137, 696

104.F. London, Z. Phys., 1932, 74, 143.

105.J. Horiuti and M. Polanyi, J. Molec. Catalysis A, 2003, 199, 185. Translation of Acta Physicochimica U.R.S.S. 1935, 2, 505532.

25 106.R. A. Marcus, Trans. N. Y. Acad. Sci., 1957, 19, 423.

107. N. S. Hush, J. Chem. Phys., 1958, 28, 962.

108.R. A. Marcus, Discuss. Faraday Soc., 1960, 21

109.N. S. Hush, Trans. Farad. Soc., 1961, 57, 577.

110.S. B. Piepho, E. R. Krausz and P. N. Schatz, J. Am. Chem. Soc., 1978, 100, 2996.

111.R. A. Marcus and N. Sutin, Biochim. Biophys. Acta, 1985, 811, 265

112.M. K. Johnson, R. B. King, J. Donald M. Kurtz, Charles Kutal, M. L. Norton and R. A. Scott, eds., Electron Transfer in Biology and the Solid State, American Chemical Society, Washington DC, 1989.

113.A. A. Kornyshev, M. Tosi and J. Ulstrup, eds., Electron and Ion Transfer in Condensed Media, World Scientific, Singapore, 1997.

114.S. Isied, ed., Electron Transfer Reactions, American Chemical Society, Washington DC, 1997.

115.A. Kutnetsov and J. Ulstrup, eds., Electron Transfer in Chemistry and Biology, Wiley, Hoboken NJ, 1999.

116.V. Balzani, ed., Electron Transfer in Chemistry. Principles, Theories, Methods and Techniques, Wiley-VCH, Hoboken NJ, 2001.

45 117.A. Nitzan, Annu. Rev. Phys. Chem., 2001, 52, 681.

118.D. M. Guldi, Chem. Soc. Rev., 2002, 31, 22.

119.T. W. Marin, B. J. Homoelle, K. G. Spears, J. T. Hupp and L. O. Spreer, J. Phys. Chem. A, 2002, 106, 1131.

120.E. A. Plummer and J. I. Zink, Inorg. Chem., 2006, 45, 6556.

50 121.S. F. Nelsen, Adv. Phys. Org. Chem., 2006, 41, 183.

122.V. Coropceanu, J. Cornil, D. A. da Silva Filho, Y. Olivier, R. Silbey and J. L. Brédas, Chem. Rev., 2007, 107, 926.

123.Z. Vager, Chem. Phys. Lett., 1997, 273, 407.

124.J. Trost and K. Hornberger, Phys. Rev. Lett., 2009, 103, 023202.

55 125.I. B. Bersuker, Chem. Rev., 2001, 101, 1067.

126.D. G. Truhlar and C. A. Mead, Phys. Rev. A, 2003, 68, 032501.

127.A. Toro-Labbe, J. Phys. Chem. A, 1999, 103, 4398.

128.P. Jaque, A. Toro-Labbe, P. Politzer and P. Geerlings, Chem. Phys. Lett., 2008, 456, 135.

60 129.R. Englman, The Jahn-Teller Effect in Molecules and Crystals, Wiley, New York, 1972.

130.G. Fischer, Vibronic Coupling, Academic Press, London, 1984

131.H. Köuppel, W. Domcke and L. S. Cederbaum, Adv. Chem. Phys., 2007, 57, 59.

65 132.K. Pae and V. Hizhnyakov, J. Chem. Phys., 2013, 138, 104103.

133.H. Rongsheng, L. Zijing and W. Kelin, Physical Review B, 2002, 65, 174303.

134.A. M. Kuznetsov, M. D. Vigdorovich and J. Ulstrup, Chem. Phys., 1993, 176, 539

70 135.A. Teklos and S. S. Skourtis, Chem. Phys., 2005, 319, 52.

136.S. S. Skourtis, D. H. Waldeck and D. N. Beratan, Annu. Rev. Phys. Chem., 2010, 61, 461.
137.J. N. Onuchic, D. N. Beratan and J. J. Hopfield, J. Phys. Chem. 1986, 90, 3707

75 138.J. Jortner, J. Chem. Phys., 1976, 64, 4860.

139.A. Warshel, J. Phys. Chem., 1982, 86, 2218

140.J. R. Reimers, L. McKemmish, R. H. McKenzie and N. S. Hush, Phys. Chem.Chem. Phys., 2015, submitted, Paper 1.

141.A. M. Wodtke, J. C. Tully and D. J. Auerbach, Int. Rev. Phys. Chem., 2004, 23, 513.

142.L. K. McKemmish, R. H. McKenzie, N. S. Hush and J. R. Reimers, J. Chem. Phys., 2011, 135, $244110 / 1$.

143.J. R. Reimers, B. B. Wallace and N. S. Hush, Phil. Trans. Roy. Soc. A, 2008, 366, 15 .

85 144.N. S. Hush, in Mechanistic Aspects of Inorganic Reactions, eds. D. B. Rorabacher and J. F. Endicott., 1982, vol. 198, pp. 301.

145.S. Woitellier, J. P. Launay and C. W. Spangler, Inorg. Chem., 1989, $28,758$.

146.J. R. Reimers and N. S. Hush, Inorg. Chem., 1990, 29, 3686.

90 147.J. R. Reimers and N. S. Hush, J. Am. Chem. Soc., 2004, 126, 4132.

148.J. F. Stanton, J. Chem. Phys., 2010, 133, 174309.

149.M. Brinkmann, G. Gadret, M. Muccini, C. Taliani, N. Masciocchi and A. Sironi, Journal of the American Chemical Society, 2000, 122, 5147.

95 150.S.-H. Lee, A. G. Larsen, K. Ohkubo, Z.-L. Cai, J. R. Reimers, S Fukuzumi and M. J. Crossley, Chem. Sci., 2011, 3, 257.

151.D. Curiel, K. Ohkubo, J. R. Reimers, S. Fukuzumi and M. J. Crossley, Phys. Chem. Chem. Phys., 2007, 9, 5260.

152.J. R. Reimers, Z.-L. Cai and H. S. Hush, Chem. Phys., 2005, 319, 39.

100 153.J. R. Reimers, W. A. Shapley, A. P. Rendell and N. S. Hush, J. Chem. Phys., 2003, 119, 3249.

154.R. Kubo and Y. Toyozawa, Prog. Theor. Phys., 1955, 13, 160.

155.V. G. Levich and R. R. Dogonadze, Dokl. Acad. Nauk. SSSR Ser. Fiz. Khim., 1959, 124, 123.

105 156.L. McKemmish, R. H. McKenzie, N. S. Hush and J. R. Reimers, Phys. Chem.Chem. Phys., 2015, submitted, Paper 4

157.E. B. D. Wilson, J. C; Cross, Paul C., Molecular Vibrations: The Theory of Infrared and Raman Vibrational Spectra, McGrawHill Book Company, New York, 1955.

110 158.R. L. Fulton and M. Gouterman, J. Chem. Phys., 1961, 35, 1059.

159.R. L. Fulton and M. Gouterman, J. Chem. Phys., 1964, 41, 2280.

160.H. Köppel, W. Domcke and L. S. Cederbaum, Adv. Chem. Phys., 1984, 57, 59

161.E. B. Wilson, Jr, J. C. Decius and P. C. Cross, Molecular Vibrations, Dover, New York, 1955.

162.N. S. Hush, Chem. Phys., 1975, 10, 361.

163.M. A. Nielsen and I. L. Chuang, Quantum Computation and Quantum Information, Cambridge University Press, New York, 2000.

120 164.J. R. Reimers, L. McKemmish, R. H. McKenzie and N. S. Hush, Phys. Chem.Chem. Phys., 2015, submitted, Paper 2.

165.S. Yin, L. Li, Y. Yang and J. R. Reimers, J. Phys. Chem. C, 2012 , 116, 14826.

166.N. S. Hush, in Proceedings of the 4th Moscow Conference on

125 Electrochemstry 1956, English translation: Consultants Bureau, New York, 1961, p. 99.

167.N. S. Hush, Z. Elektrochem. Angewandte Physik. Chem., 1957, 61, 734.

168.N. S. Hush, Disc. Farad. Soc., 1960, 29, 113

130 169.N. S. Hush, Prog. Inorg. Chem., 1967, 8, 391.

170.S. C. Cheng, C. Zhu, K. K. Liang, S. H. Lin and D. G. Truhlar, J. Chem. Phys., 2008, 129, 024112.

171.Y. Arasaki, K. Takatsuka, K. Wang and V. McKoy, Phys. Rev. Lett., 2003, 90, 248303.

135 172.H. Nakamura, J. Phys. Chem. A, 2006, 110, 10929.

173.G. Nan, L. Wang, X. Yang, Z. Shuai and Y. Zhao, J. Chem. Phys., 2009, 130, 024704/1.

174.G. Nan, Q. Shi, Z. Shuai and Z. Li, Phys. Chem. Chem. Phys., 2011 13, 9736 .

140 175.B. R. Landry and J. E. Subotnik, J. Chem. Phys., 2012, 137, 22A513.

176.E. J. Heller, Acc. Chem. Res., 1981, 14, 368.

177.A. W. Jasper and D. G. Truhlar, J. Chem. Phys., 2005, 123, 064103.

178.H. Han and P. Brumer, J. Chem. Phys., 2005, 122, 144316.

179.O. V. Prezhdo and P. J. Rossky, J. Chem. Phys., 1997, 107, 5863.

145 180.N. S. Hush, Electrochim. Acta, 1968, 13, 1005 
181.S. Glasstone, K. J. laidler and H. Eyring, The Theory of rate processes, McGraw Hill, New York, 1941.

182.B. S. Brunschwig, J. Logan, M. D. Newton and N. Sutin, J. Am. Chem. Soc., 1980, 102, 5798

5 183.J. E. Norton and J. L. Brédas, J. Am. Chem. Soc., 2008, 130, 12377.

184.A. Abedi, N. T. Maitra and E. K. U. Gross, Phys. Rev. Lett., 2010, $\mathbf{1 0 5}, 123002$.

185.A. Abedi, N. T. Maitra and E. K. U. Gross, J. Chem. Phys., 2012, $137,22 \mathrm{~A} 530$

10 186.L. S. Cederbaum, J. Chem. Phys., 2013, 138, 224110.

187.G. Hunter, Int. J. Quantum Chem., 1975, 9, 237.

188.J. Pu, J. Gao and D. G. Truhlar, Chem. Rev., 2006, 106, 3140

189.A. M. Stoneham, Theory of defects in solids, Oxford University Press, Oxford, 1975.

15 190.R. A. Marcus, J. Chem. Phys., 1965, 43, 679.

191.R. A. Marcus, J. Chem. Phys., 1956, 24, 966.

192.R. A. Marcus, Annu. Rev. Phys. Chem., 1964, 15, 155. 
Cite this: DOI: 10.1039/coxxooooox

\section{TOC graphic}

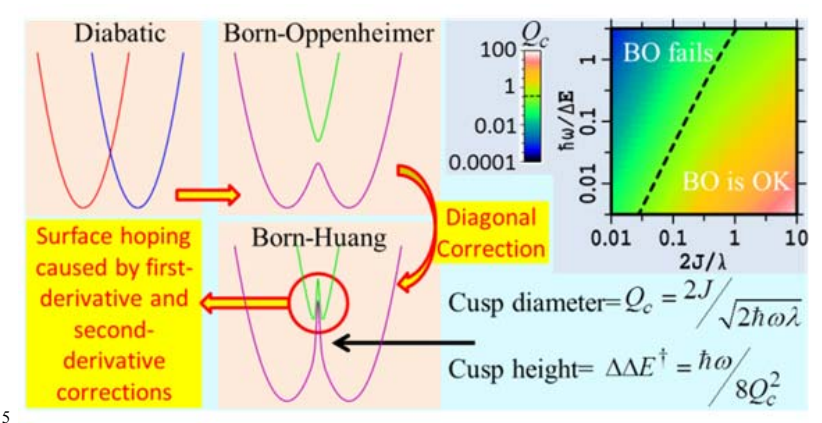

